\title{
Delay-Throughput Tradeoff with Correlated Mobility of Ad-Hoc Networks
}

\author{
Shuochao Yao*, Xinbing Wang*, Xiaohua Tian*, Qian Zhang ${ }^{\dagger}$ \\ ${ }^{*}$ Dept. of Electronic Engineering, Shanghai Jiao Tong University, China \\ ${ }^{\dagger}$ Dept. of Computer Science and Engineering, Hong Kong University of Science and Technology, China \\ \{sasukecao,xwang8,xtian\}@sjtu.edu.cn, qianzh@cse.ust.hk
}

\begin{abstract}
We further the analysis of the scaling law in wireless ad hoc networks with the correlated mobility model. The former work about correlated mobility has shown the maximum throughput and the corresponding delay of several sub-cases, but the optimal throughput performances under various delay tolerant condition (the optimal delay-throughput tradeoff) remains open. We study the properties of correlated mobility model and establish the overall upper bound of delay-throughput tradeoff. Then we find out an achievable lower bound by studying the optimal scheduling parameters and their constrains. We exploit the node correlation to achieve the delay-throughput tradeoff and give a relatively whole picture that how node correlation impacts the packet delay, asymptotic throughput, and their tradeoff.
\end{abstract}

\section{INTRODUCTION}

Since the breakthrough work by Gupta and Kumar [1], people have shown great interest in the network capacity for large scale wireless ad hoc networks. Gupta and Kumar show us that the per-node throughput can only achieve $O(1 / \sqrt{n \log n})$ as the number of nodes $n$ increases in a static network. Then Franceschetti and Dousse [2] show that the $O(1 / \sqrt{n})$ per-node throughput is achievable by applying percolation theory, but it is still a pessimistic conclusion for that the network capacity decay rapidly as number of node $n$ increase.

In a seminal work [3], Grossglauser and Tse show that the per-node throughput can achieve $O(1)$ when mobility takes into account, however the cost of improvement in per-node throughput is the unbounded delay. So studies begin to focus on revealing the relationship between the per-node throughput and the packet delay (delay-throughput tradeoff). Neely et al. [4] study the fast mobility with i.i.d. model. Toumpis et al. [5], and Lin et al. [6] study the slow mobility with i.i.d. model. They show us the impact of i.i.d. mobility on the delaythroughput tradeoff.

Several works analyse the delay-throughput tradeoff for different mobility models because the details of how nodes mobile play a role in the tradeoff. From Gamal et al. [7] for the Brownian motion mobility model, to Bansal et al. [8] for the random waypoint model, Diggavi et al. [9] for the linear mobility model, [10], [11], [12] for the restricted mobility model, and Ciullo et al. [13] for the correlated mobility model.

Mobility of nodes in [7] and [8] is uniform over the network area and uncorrelated (i.e., nodes are independent from each other). Mobility of nodes in [10]- [12] introduces the nonuniform condition, but it is still uncorrelated. The real mobilities show high degree of correlation [14], [15], [16],
[17], so our work focus on the impact of correlated mobility on the delay-throughput tradeoff of ad hoc network.

Correlated mobility can be divided into three sub-cases according to the node correlation: the cluster sparse regime (nodes show strong correlation), the cluster dense regime (nodes show weak correlation), and the cluster critical regime (nodes show medium correlation). Ciullo et al. [13] presents a fine opening for the study of correlated mobility. They show the optimal maximum throughput with the corresponding packet delay under cluster sparse regime and the lower bound of maximum throughput with the corresponding packet delay under cluster dense regime. The optimal throughput performances under various delay tolerant condition (not only the maximum one) are important for the applications require for different packet delays, and it is also a hard problem to establish and formulate the optimal redundance creating and messages forwarding scheme because of the various network topologies of different node correlations. So we study the following open question in this paper:

- What is the optimal delay-throughput tradeoff with correlated mobility (for all sub-cases) of ad hoc network?

The goal of our work is to address the question above. We study the properties of the correlated mobility and establish an upper bound on the optimal delay-throughput tradeoff in mobile ad-hoc network with correlated mobility (for three subcases). Further, we develop a scheduling policy that achieve the upper bound of throughput-delay tradeoff up to a logarithmic factor. During our analysis, a novel double scheduling policy and "inter-cluster duplication" are specially designed for various degree of node correlation.

According to the delay-throughput tradeoff we have deduced, we have the following observation about the correlated mobility. 1) cluster sparse regime suffers the constraints of maximum throughput and minimum delay, because extremely strong correlation among nodes causes network disconnectivity. 2) cluster dense and critical regimes perform better than (at least equal to) the i.i.d. model. 3) Neither strong nor extremely weak node correlation helps improving the network performance. The main contribution of our work is that we are the first to exploit the node correlation in order to give a relatively whole picture that how node correlation impacts the delay-throughput tradeoff, and show how to control the impact through system and scheduling parameters.

The rest of paper is organized as follows. In section II, we 
introduce our network and mobility model. In section III, we establish the upper bound of cluster sparse regime. In section IV, we show the detailed upper bound of cluster sparse regime by using the optimal scheduling parameters. In section V, we present an achievable lower bound of cluster sparse regime. In section VI, we establish the upper bound of cluster dense regime. In section VII, we show the achievable lower bound of cluster dense regime. In section VIII and section IX, we give the upper bound and the achievable lower bound of cluster critical regime respectively. In section X, we discuss our delaythroughput tradeoff of correlated mobility. In section XI, we conclude.

\section{Network ANd Mobility Model}

\section{A. Network Topology}

We consider $n$ nodes moving over a square with area $n$, and nodes are divided into $m=\Theta\left(n^{v}\right)(0 \leq v<1)$ groups. Each group covers a circular area with radius $R=\Theta\left(n^{\beta}\right)(0 \leq$ $\beta \leq 1 / 2)$. In the following section, we will refer such groups as clusters. We assume each group contains $q=n / m$ nodes and the our result won't change if the number of nodes each cluster contains is not exactly the same but remains $\Theta(m / n)$. The definitions of these system parameters are shown in Table I.

We assume time is divided into time slots of unit duration. The positions of nodes are static during each time slot and nodes mobile with correlated fashion between each time slot. We describe the mobility of node $i$ in cluster $j$ with two steps: 1) At the begining of each time slot, the position of cluster $j$ 's center is i.i.d. and uniformly chosen among the whole network area at random, independently from other clusters; 2) Then the position of node $i$ is i.i.d. and uniformly chosen among the circular area that cluster $j$ covers at random, independently from other nodes in cluster $j$. The above two steps are called group movement and node movement respectively; the combination of them describes the correlated mobility in our work. We observe that nodes show strong correlation if we either reduce the number of clusters $m$ (i.e., smaller value of $v$ ) or reduce the area each cluster covers (i.e., smaller value of $\beta$ ).

\section{B. Transmission Protocol}

To limit the interference, we adopt the protocol model proposed in [1]. Let $X_{i}$ denote the position of node $i$ ( $i=$ $1, \ldots, n)$ and $\left|X_{i}-X_{j}\right|$ denote the Euclidean distance between node $i$ and $j$. A transmitter $i$ can transmit at $W$ bit/second successfully to a destination $j$ when

$$
\left|X_{j}-X_{k}\right| \geq(1+\Delta)\left|X_{i}-X_{j}\right|
$$

for any other simultaneously active transmitters $k$, where $\Delta$ is a positive number.

Slow mobility is considered in our work, which means multihop schedule can be operated within a single time slot. Situation for fast mobility can be extended from our analysis. Moreover, we consider transmission among different clusters,
TABLE I: System Parameter.

\begin{tabular}{|l|l|}
\hline$n$ & number of nodes \\
\hline$m$ & number of cluster \\
\hline$v$ & growth exponent of $m: m=\Theta\left(n^{v}\right), 0<v \leq 1$ \\
\hline$q$ & average number of node per cluster, $q=n / m=\Theta\left(n^{1-v}\right)$ \\
\hline$R$ & cluster radius \\
\hline$\beta$ & growth exponent of $R: R=\Theta\left(n^{\beta}\right)$ \\
\hline
\end{tabular}

because the transmission within the same cluster is a kind of i.i.d. model which has been discussed in many former works.

\section{Traffic Model}

We assume all sources communicate with their destinations at same rate $\lambda$ and $\bar{D}$ denote the average delay over all messages among all source-destination pairs.

Definition of Asymptotic Throughput and Delay: Let $\lambda_{i}$ $(i=1, \ldots, n)$ denote the sustainable rate of data flow for node $i$ and $D_{b}(b=1, \ldots, \lambda n T)$ denote the sustainable data delay for message $b$. Assume that $\lambda=\min \left\{\lambda_{1}, \lambda_{2}, \ldots, \lambda_{n-1}, \lambda_{n}\right\}$ and $\bar{D}=\sum_{b=1}^{\lambda n T} D_{b} / \lambda n T$. Then $\lambda=\Theta(f(n))$ is defined as the asymptotic throughput if there exist constant $c>c^{\prime}>0$, that

$$
\begin{aligned}
& \lim _{n \rightarrow \infty} \operatorname{Pr}(\lambda=c f(n) \text { is achievable })<1, \\
& \lim _{n \rightarrow \infty} \operatorname{Pr}\left(\lambda=c^{\prime} f(n) \text { is achievable }\right)=1 .
\end{aligned}
$$

And $\bar{D}=\Theta(g(n))$ is defined as the asymptotic delay as well.

\section{Upper Bound of the Cluster Sparse Regime}

We divide our model into three sub-cases: the cluster sparse regime when $v+2 \beta<1$ (i.e., $m R^{2}=o(n)$, strong node correlation), the cluster dense regime when $v+2 \beta>1$ (i.e., weak node correlation), and the cluster critical regime when $v+2 \beta=1$ (i.e., medium node correlation). In this section we consider the upper bound of delay-throughput tradeoff under cluster sparse regime.

Under cluster sparse regime, $m$ clusters only cover a negligible fraction of whole network area. In the other aspect, density in the cluster is relatively high (the density is about $\left.n /\left(m R^{2}\right)=\omega(1)\right)$ and overlaps between different clusters are sporadic, which indicate strong node correlation.

\section{A. Scheduling policy}

In this section, we will first design a scheduling policy, referring to some scheduling parameters. Then we propose several lemmas to exclude the parameters not affecting the asymptotic throughput and delay.

For a traffic stream $s \rightarrow d$ (where $s$ denotes the source and $d$ denotes the destination), we denote $C_{s}$ as the cluster containing $s$ and $C_{d}$ as the cluster containing $d$. We assume $C_{s} \neq C_{d}$, which maximize the character of correlated mobility. Opportunistic broadcast scheme (nodes only broadcast when existing a large number of nodes around or scheme will choose another slot to broadcast) is applied here. Our original scheduling policy is shown as follow: 


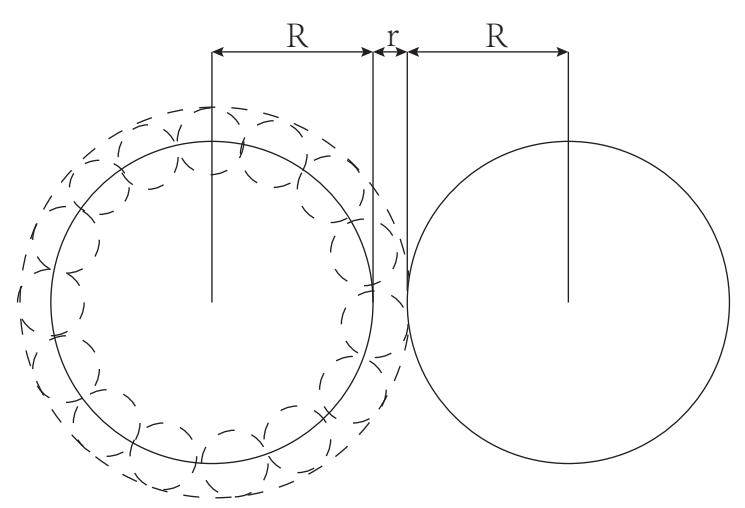

Fig. 1: Transmission between two clusters.

1) $s$ creates $R_{s}$ relays in $C_{s}$ via muticast.

2) When a relay meets a cluster $C_{k}\left(k=1, \ldots, R_{c}{ }^{s}\right.$, where $R_{c}{ }^{s}$ is the maximum number of clusters containing relays under cluster sparse regime) not containing a relay, a relay will be created in $C_{k}$ via one-hop unicast.

3) New-created relay in $C_{k}$ creates $R_{k}$ relays in $C_{k}$ via broadcast.

4) If a relay meets $C_{d}$, a new relay will be created in $C_{d}$ via one-hop unicast. If not, we come back to step 2).

5) Relays in $C_{d}$ create new relays in $C_{d}$ via broadcast $\left(R_{d}{ }^{s}\right.$ denotes the overall number of relays in $C_{d}$ ).

6) If one of $R_{d}^{s}$ relays is captured by the destination with range $l^{s}$, the message will be transmitted to destination via $h^{s}$-hop unicast. If not, we come back to step 5).

Our policy can be divided into two parts: forwarding messages form $C_{s}$ to $C_{d}$ and forwarding messages within $C_{d}$. The network topology of the second part is similar as i.i.d. model, so we focus on the first part which reflects the character of correlated mobility.

In the first part, we use term "inter-cluster duplication" to denote the cluster containing relays; we use term "intracluster duplication" to denote the relays in a certain cluster. In our original policy, the number of inter-cluster duplications is $R_{c}{ }^{s}$, and the number of intra-cluster duplications is a set $\left\{R_{s}, R_{1}, \ldots, R_{R_{c}}{ }, R_{d}\right\}$. As radio resource is needed to create relays, Lemma 3.1 will help us to simplify the schedule.

Lemma 3.1: Under cluster sparse regime, most intra-cluster duplications $\left\{R_{s}, R_{1}, \ldots, R_{R_{c}}\right\}$ will decrease the asymptotic throughput without decreasing the asymptotic delay.

We will give detailed proof of Lemma 3.1 in Lemma 6.2. Now we just let it as something we have already proved. So $R_{k}=1$ for $k=s, 1,2, \ldots, R_{c}{ }^{d}$. We can particularly use "intra-cluster duplication" to denote relays in $C_{d}$. Then our scheduling policy is show below, and we illustrate the scheduling policy in Fig. 2. Opportunistic broadcast scheme (nodes only broadcast when existing a large number of nodes around or scheme will choose another slot to broadcast) is applied here.

1) When $s$ and relays meet a cluster $C_{k}\left(k=1, \ldots, R_{c}{ }^{s}\right.$, where $R_{c}{ }^{s}$ is the maximum number of inter-cluster duplications) not containing a relay, a relay will be created in $C_{k}$ via one-hop unicast.

2) If a relay meets $C_{d}$, a relay will be created in $C_{d}$ via one-hop unicast. If not, we come back to step 1).

3) Relays in $C_{d}$ create intra-cluster duplications in $C_{d}$ via broadcast $\left(R_{d}^{s}\right.$ denotes the overall number of intracluster duplications).

4) If one intra-cluster duplication is captured by the destination with range $l^{s}$, the message will be transmitted to destination via $h^{s}$-hop unicast. If not, we come back to step 3)

\section{B. Tradeoff for delay}

This section will show us a fundamental tradeoff about delay, which is one of the cornerstones for deriving the upper bound of delay-throughput tradeoff. First we will give an intuitive explanation; then we give the analysis for sophisticated strategy shown above.

Our scheduling policy can be divided into three parts. $D_{I}^{s}$ denotes the delay of creating $R_{c}{ }^{s}$ inter-cluster duplications, $D_{I I}^{s}$ denotes the delay of transmission from $R_{c}{ }^{s}$ inter-cluster duplications to $C_{d}$, and $D_{I I I}^{s}$ denotes the delay of transmission within $C_{d}$.

As for $D_{I}^{s}$, we assume that $D_{I}^{s}=\sum_{k=1}^{R_{c}{ }^{s}} D_{I k}^{s}$, where $D_{I k}^{s}$ stands for the delay of creating the $k$ th inter-cluster duplication. We denote $\mathbf{P}_{\mathbf{I}}^{\mathbf{s}}(k)$ as the probability that intercluster duplications meet a cluster not containing a relay, when we have already created $k-1$ inter-cluster duplications.

From Fig. 1, we can obtain that

$$
\mathbf{P}_{\mathbf{I}}^{\mathbf{s}}(k)=1-\left(1-\frac{\pi k(2 R+r)^{2}}{n}\right)^{m-k}
$$

Then it's easy to obtain $D_{I k}^{s}=1 / \mathbf{P}_{\mathbf{I}}^{\mathbf{s}}(k)$, which leads to

$$
\begin{aligned}
D_{I}^{s} & =\sum_{k=1}^{R_{c}{ }^{s}} \frac{1}{1-\left(1-\pi k(2 R+r)^{2} / n\right)^{m-k}} \\
& \geq \sum_{k=1}^{R_{c}{ }^{s}} \frac{n}{\pi k(m-k)(2 R+r)^{2}} \\
& =\frac{n}{\pi m(2 R+r)^{2}} \sum_{k=1}^{R_{c}{ }^{s}} \frac{1}{k}+\frac{1}{m-k} \\
& \geq \Theta\left(\frac{n}{m R^{2}}\left(\ln \frac{R_{c}{ }^{s} m}{m-R_{c}{ }^{s}}+\gamma\right)\right)
\end{aligned}
$$

where $\gamma$ is the Euler constant and $r$ is the transmission range for a single hop.

As for $D_{I I}^{k}$, the delay of forwarding a message from $R_{c}$ inter-cluster duplications to $C_{d}$ can be formulated by

$$
\begin{aligned}
D_{I I}^{s} & =\frac{1}{1-\left(1-\pi(R-r)^{2} / n\right)^{R_{c}{ }^{s}}} \\
& \geq \Theta\left(\frac{n}{R_{c}^{s} R^{2}}\right)
\end{aligned}
$$

Similarly,

$$
D_{I I I}^{s} \geq \Theta\left(\frac{R^{2}}{R_{d}^{s} l^{2}}\right)
$$



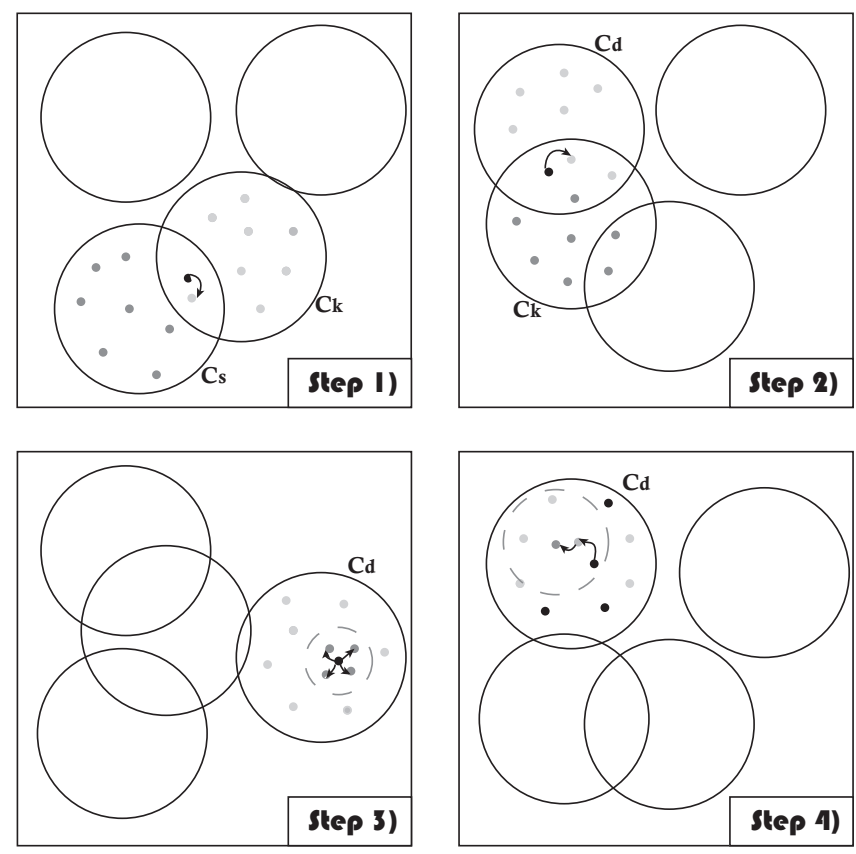

Fig. 2: Scheduling policy under cluster sparse regime.

Considering these three delays $D_{I}^{s}, D_{I I}^{s}$, and $D_{I I I}^{s}$, the total delay $D^{s}=\max \left\{D_{I}^{s}, D_{I I}^{s}, D_{I I I}^{s}\right\}$, but $D_{I \max }^{s} \geq$ $\Theta\left(n \log m / m R^{2}\right)$ and $D_{I I \min }^{s} \geq \Theta\left(n / m R^{2}\right)$, which means $D_{I}^{s}$ will not exceed $D_{I I}^{s}$ by a logarithmic factor when considering the bound. Omitting the logarithmic factor, we obtain

$$
D_{b}^{s}=\max \left\{D_{I I b}^{s}, D_{I I I b}^{s}\right\}
$$

where $b$ stands for a particular bit.

A more sophisticated strategy (which is our schedule shown in the former section) is "opportunistic duplication scheme". A message will be transmitted to its target from the relays, if one of the relays obtains a chance to communicate with target at each time slot $t$. Otherwise, duplications will be created as normal. This scheme may obtain a better result for $D_{I}^{s}+$ $D_{I I}^{s}$ and $D_{I I I}^{s}$, however the following lemma shows that this scheme can only improve the delay with a $\log n$ factor.

Lemma 3.2: Under the cluster sparse regime, the delay for a particular bit $b$ and its scheduling parameters comply the following inequality

$$
c_{1}^{s} \log n \mathbb{E}\left[D_{b}^{s}\right] \geq \max \left\{\frac{n}{R^{2} \mathbb{E}\left[R_{c b}^{s}\right]}, \frac{R^{2}}{\mathbb{E}\left[R_{d b}^{s}\right] \mathbb{E}\left[l_{b}^{s}+\frac{m R^{2}}{n^{2}}\right]^{2}}\right\}
$$

where $c_{1}^{s}$ is a positive constant and variable $X_{b}^{s}$ denotes the variable $X$ under cluster sparse regime for a particular bit $b$.

The proof of Lemma 3.2 is shown in Appendix A.

\section{Tradeoff for radio resource}

This section will show us another fundamental tradeoff about radio resource. Firstly, we will recall the disjoint disk. Then we will focus on some special properties under cluster sparse regime. At last, we will obtain the tradeoff between radio resource and related scheduling parameters.
We use protocol model as our communication model, so disjoint disk is a specific model describing limited radio resource, which is first proved in [1].

Consider that nodes $i, j$ directly transmit to nodes $k$ and $l$ respectively, at the same time. Then, according to the interference constraint:

$$
\begin{array}{r}
\left|X_{j}-X_{k}\right| \geq(1+\Delta)\left|X_{i}-X_{k}\right| \\
\left|X_{i}-X_{l}\right| \geq(1+\Delta)\left|X_{j}-X_{l}\right|
\end{array}
$$

Hence,

$$
\begin{aligned}
\left|X_{j}-X_{i}\right| & \geq\left|X_{j}-X_{k}\right|-\left|X_{i}-X_{k}\right| \\
& \geq \Delta\left|X_{i}-X_{k}\right|
\end{aligned}
$$

Therefore,

$$
\left|X_{j}-X_{i}\right| \geq \frac{\Delta}{2}\left(\left|X_{i}-X_{k}\right|+\left|X_{j}-X_{l}\right|\right)
$$

disks of radius $\Delta\left|X_{i}-X_{k}\right| / 2$, where $i, k$ is a sending-receiving pair, centering at sender are disjoint from each other.

Under the cluster sparse cluster, nodes only cover a small part of network area at each time slot. This phenomenon leads to two properties we need to notice as we deduce the tradeoff.

One is that the area of radio resource we use is only $\Theta\left(m R^{2}\right)$, not $\Theta(n)$ as the i.i.d. model. The other is that [13] have proved that a certain cluster has only a probability of $m R^{2} / n$ to meet other clusters. When creating a intercluster duplication, $n /\left(m R^{2}\right)$ chances are needed to ensure a successful operation. Creating $R_{c b}{ }^{s}$ inter cluster duplications is equivalent to $n R_{c b}^{s} /\left(m R^{2}\right)$ times one-hop unicast.

Lemma 3.3: Under cluster sparse regime and concerning radio resource, the throughput for a particular bit $b$ and its scheduling parameters comply the following inequality

$$
\sum_{b=1}^{\lambda^{s} n T} \frac{\Delta^{2}}{4} \frac{\mathbb{E}\left[R_{d b}^{s}\right]-1}{n}+\mathbb{E}\left[\sum_{b=1}^{\lambda^{s} n T} \sum_{h=1}^{h_{b}^{s}+\frac{n R_{c b}^{s}}{m R^{2}}} \frac{\pi \Delta^{2}}{4} \frac{r_{b}^{h^{2}}}{m R^{2}}\right] \leq c_{2}^{s} W T \log n
$$

where $c_{2}^{s}$ is a positive number, $h_{b}^{s}$ is the number of transmission hops after message being captured by the destination, and $r_{b}^{h}$ is the transmission range of each hop, $h=1, \ldots, h_{b}^{s}$. Opportunistic broadcast scheme (nodes only broadcast when existing a large number of nodes around) is applied here.

Proof is similar to Appendix B in [6], so we omit it for simplification.

\section{Tradeoff for Half Duplex and Mutihop}

Since no node can transmit and receive at the same time and over same frequency, the following inequality holds,

Lemma 3.4: The following inequality holds,

$$
\sum_{b=1}^{\lambda^{s} n T} \sum_{h=1}^{h_{b}^{s}+\frac{n R_{c} s}{m R^{2}}} 1 \leq \frac{W T}{2} n
$$

The following inequality holds for the nature of multihop. Lemma 3.5: The following inequality holds,

$$
\sum_{b=1}^{\lambda^{s} n T} \sum_{h=1}^{h_{b}^{s}} r_{b}^{h} \geq l_{b}^{s}
$$




\section{E. Upper bound on delay-throughput tradeoff}

The upper bound under cluster sparse regime can be derived from the basic tradeoffs we have proven. In this section, we will separate our proof into two parts. One is $D_{I I I}^{s} \geq D_{I I}^{s}$ and the other is $D_{I I I}^{s}<D_{I I}^{s}$.

Lemma 3.6: Under cluster sparse regime, when $D_{I I I}^{s} \geq$ $D_{I I}^{s}$, let $\overline{D^{s}}$ denote the mean delay averaged over all bits and let $\lambda^{s}$ be the throughput of each source-destination pair. The following upper bound holds,

$$
\left(\lambda^{s}\right)^{3} \leq O\left(\frac{m \bar{D}^{s}}{n} \log ^{3} n\right)
$$

Proof: From Lemma 3.2, when $D_{I I I}^{s} \geq D_{I I}^{s}$, we have

$$
\begin{aligned}
c_{1}^{s} \log n \mathbb{E}\left[D_{b}^{s}\right] \geq & \frac{R^{2}}{\mathbb{E}\left[R_{d b}^{s}\right]\left(\mathbb{E}\left[l_{b}^{s}\right]+\frac{m R^{2}}{n^{2}}\right)^{2}} \\
\sum_{b=1}^{\lambda^{s} n T} \mathbb{E}\left[R_{d b}^{s}\right] \geq & \frac{1}{c_{1}^{s} \log n} \sum_{b=1}^{\lambda^{s} n T} \frac{R^{2}}{\left(\mathbb{E}\left[l_{b}^{s}\right]+\frac{m R^{2}}{n^{2}}\right)^{2} \mathbb{E}\left[D_{b}^{s}\right]} \\
\geq & \frac{R^{2}}{c_{1}^{s} \log n} \frac{\sum_{b=1}^{\lambda^{s} n T} 1}{\sum_{b=1}^{\lambda^{s} n T} \mathbb{E}\left[D_{b}^{s}\right]} \\
& \times \frac{\left(\sum_{b=1}^{\lambda^{s} n T} 1\right)^{3}}{\left(\sum_{b=1}^{\lambda^{s} n T}\left(\mathbb{E}\left[l_{b}^{s}\right]+\frac{m R^{2}}{n^{2}}\right)\right)^{2}} \\
= & \frac{\left(R^{2}\right.}{c_{1}^{s} \log n} \frac{\left(\sum_{b=1}^{\lambda^{s} n T} 1\right)^{3}}{\overline{D^{s}}\left(\sum_{b=1}^{\lambda^{s} n T}\left(\mathbb{E}\left[l_{b}^{s}\right]+\frac{m R^{2}}{n^{2}}\right)\right)^{2}}
\end{aligned}
$$

Inequality (6) is deduced by using Jensen's Inequality and Hölder's Inequality. From Lemma 3.3 and Cauchy-Schwartz inequality, we obtain

$$
\begin{aligned}
& \frac{\pi \Delta^{2}}{2 W T n m R^{2}}\left(\sum_{b=1}^{\lambda^{s} n T} \mathbb{E}\left[\sum_{h=1}^{h_{b}^{s}+\frac{n R_{c b}^{s}}{m R^{2}}} r_{b}^{h}\right]\right)^{2} \\
& \quad+\sum_{b=1}^{\lambda^{s} n T} \frac{\Delta^{2}}{4} \frac{\mathbb{E}\left[R_{d b}^{s}\right]}{n}-\frac{\Delta^{2}}{4} \lambda^{s} T \leq c_{2}^{s} W T \log n
\end{aligned}
$$

Case 1: when $h_{b}^{s} \geq \frac{n R_{c_{b}}^{s}}{m R^{2}}$, then

$$
\begin{gathered}
\sum_{b=1}^{\lambda^{s} n T} \frac{\Delta^{2}}{4} \frac{\mathbb{E}\left[R_{d b}^{s}\right]}{n}+\frac{\pi \Delta^{2}}{2 W T n m R^{2}}\left(\sum_{b=1}^{\lambda^{s} n T} \mathbb{E}\left[l_{b}^{s}\right]\right)^{2} \leq c_{2}^{s} W T \log n \\
\frac{\Delta^{2} R^{2}}{4 c_{1}^{s} n \log n} \frac{\left(\sum_{b=1}^{\lambda^{s} n T} 1\right)^{3}}{\bar{D}^{s}\left(\sum_{b=1}^{\lambda^{s} n T}\left(\mathbb{E}\left[l_{b}^{s}\right]+\frac{m R^{2}}{n^{2}}\right)\right)^{2}} \\
+\frac{\pi \Delta^{2}}{2 W T n m R^{2}}\left(\sum_{b=1}^{\lambda^{s} n T} \mathbb{E}\left[l_{b}^{s}\right]\right)^{2} \leq c_{2}^{s} W T \log n \\
\text { If } \sum_{b=1}^{\lambda^{s} n T}\left[l_{b}^{s}\right]<\lambda^{s} m R^{2} T / n, \\
\frac{\Delta^{2} R^{2}}{4 c_{1}^{s} n \log n} \frac{\left(\lambda^{s} n T\right)^{3} n^{2}}{\bar{D}^{s}\left(\lambda^{s} m R^{2} T\right)^{2}} \leq c_{2}^{s} W T \log n \\
\frac{\Delta^{2} \lambda^{s} n^{4} T}{4 c_{1}^{s} \bar{D}^{s} m^{2} R^{2} \log n} \leq c_{2}^{s} W T \log n \\
\lambda^{s} \leq \frac{4 c_{1}^{s} c_{2}^{s} W T \bar{D}^{s} m^{2} R^{2} \log ^{2} n}{\Delta^{2} n^{4} T}
\end{gathered}
$$

$$
\text { If } \sum_{b=1}^{\lambda^{s} n T}\left[l_{b}^{s}\right] \geq \lambda^{s} m R^{2} T / n,
$$

$$
\begin{aligned}
\frac{\Delta^{2} R^{2}}{4 c_{1}^{s} n \log n} \frac{\left(\sum_{b=1}^{\lambda^{s} n T} 1\right)^{3}}{\bar{D}^{s}\left(\sum_{b=1}^{\lambda^{s} n T} \mathbb{E}\left[l_{b}^{s}\right]\right)^{2}} & \\
+\frac{\pi \Delta^{2}}{2 W T n m R^{2}}\left(\sum_{b=1}^{\lambda^{s} n T} \mathbb{E}\left[l_{b}^{s}\right]\right)^{2} & \leq c_{2}^{s} W T \log n \\
\sqrt{\frac{\pi \Delta^{2} T^{2}}{8 c_{1}^{s} W \log n} \frac{\left(\lambda^{s}\right)^{3} n}{m \bar{D}^{s}}} & \leq c_{2}^{s} W T \log n \\
\left(\lambda^{s}\right)^{3} & \leq \frac{8 c_{1}^{s}\left(c_{2}^{s}\right)^{2} W^{3} m \bar{D}^{s} \log ^{3} n_{(9)}}{\pi \Delta^{2} n}
\end{aligned}
$$

Case 2: when $h_{b}^{s} \leq \frac{n R_{c b}^{s}}{m R^{2}}$, then

Decreasing number of hops $h_{b}^{s}$ for each bit will not consume the radio resource asymptotically, however it may decrease the capture range and increase the delay. So we assume $h_{b}^{s}=\Theta\left(n R_{c b}^{s} /\left(m R^{2}\right)\right)$; all $h_{b}^{s}$ and $n R_{c b}^{s} /\left(m R^{2}\right)$ above are interchangeable when we consider asymptotic throughput and delay.

Finally we compare the two Inequalities (7) and (9). Inequality (9) is the upper bound of delay-throughput tradeoff when $D_{I I I}^{s} \geq D_{I I}^{s}$.

$$
\left(\lambda^{s}\right)^{3} \leq O\left(\frac{m \bar{D}^{s}}{n} \log ^{3} n\right)
$$

Lemma 3.7: Under cluster sparse regime, when $D_{I I I}^{s}<$ $D_{I I}^{s}$, let $\bar{D}^{s}$ denote the mean delay averaged over all bits and let $\lambda^{s}$ be the throughput of each source-destination pair. The following upper bound holds,

$$
\lambda^{s} \leq O\left(\frac{m R^{4} \bar{D}^{s}}{n^{2}} \log ^{3} n\right)
$$

Proof: From Lemma 3.2, when $D_{I I I}^{s}<D_{I I}^{s}$, we have

$$
\begin{aligned}
c_{1}^{s} \log n \mathbb{E}\left[D_{b}^{s}\right] & \geq \frac{n}{R^{2} \mathbb{E}\left[R_{c b}^{s}\right]} \\
\sum_{b=1}^{\lambda^{s} n T} \mathbb{E}\left[R_{c b}^{s}\right] & \geq \frac{1}{c_{1}^{s} \log n} \sum_{b=1}^{\lambda^{s} n T} \frac{n}{R^{2} \mathbb{E}\left[D_{b}^{s}\right]} \\
& \geq \frac{n}{c_{1}^{s} \log n R^{2}} \frac{\left(\sum_{b=1}^{\lambda^{s} n T} 1\right)^{2}}{\sum_{b=1}^{\lambda^{s} n T} \mathbb{E}\left[D_{b}^{s}\right]} \\
& =\frac{n\left(\sum_{b=1}^{\lambda^{s} n T} 1\right)}{c_{1}^{s} \log n R^{2} \bar{D}^{s}}
\end{aligned}
$$

Inequality (10) is deduced using Jensen's Inequality. From Lemma 3.3 and assume $h_{b}^{s}=n^{\gamma} n R_{c b}^{s} /\left(m R^{2}\right),\left(1 \leq h_{b}^{s} \leq\right.$ $n / m$ ) we obtain 
TABLE II: The order of optimal values of the scheduling parameters under cluster sparse regime when $D_{I I I}^{s} \geq D_{I I}^{s}$.

\begin{tabular}{|l|l|}
\hline$R_{d b}^{s}:$ \# of Intra-cluster duplications & $\Theta\left(n^{\frac{1-d-v}{3}}\right)$ \\
\hline$R_{c b}^{s}:$ \# of Inter-cluster duplications & $\Theta\left(n^{1-d-2 \beta} / \log n\right)$ \\
\hline$l_{b}^{s}:$ Capture Range & $\Theta\left(n^{\frac{v+6 \beta-2 d-1}{6}} / \log ^{\frac{1}{2}} n\right)$ \\
\hline$h_{b}^{s}:$ \# of Hops & $\Theta\left(n^{\frac{1-v-d}{3}} / \log n\right)$ \\
\hline$r_{b}^{h}:$ Transmission range of Each Hop & $\Theta\left(n^{\frac{v-1+2 \beta}{2}} \log ^{\frac{1}{2}} n\right)$ \\
\hline
\end{tabular}

$$
\begin{gathered}
\frac{\pi \Delta^{2}}{4 m n R^{2}} \sum_{b=1}^{\lambda^{s} n T} \mathbb{E}\left[\sum_{h=1}^{\frac{\left(1+n^{\gamma}\right) n R_{c b}^{s}}{m R^{2}}} n r_{b}^{h^{2}}\right] \\
+\sum_{b=1}^{\lambda^{s} n T} \frac{\Delta^{2}}{4} \frac{\mathbb{E}\left[R_{d b}^{s}\right]}{n} \leq c_{2}^{s} W T \log n \\
\frac{\pi \Delta^{2} n}{4 m^{2} R^{4}} \sum_{b=1}^{\lambda^{s} n T} \frac{\left(1+n^{\gamma}\right) \mathbb{E}\left[R_{c b}^{s} r_{b}^{h^{2}}\right]}{\log n} \\
\frac{+\sum_{b=1}^{\lambda^{s} n T} \frac{\Delta^{2}}{4} \frac{\mathbb{E}\left[R_{d b}^{s}\right]}{n} \leq 2 c_{2}^{s} W T \log n}{4 m^{2} R^{4}} \sum_{b=1}^{\lambda^{s} n T} \frac{\left(1+n^{\gamma}\right) \mathbb{E}\left[R_{c b}^{s}\right] \mathbb{E}\left[r_{b}^{h}\right]^{2}}{\log n} \\
+\sum_{b=1}^{\lambda^{s} n T} \frac{\Delta^{2}}{4} \frac{\mathbb{E}\left[R_{d b}^{s}\right]}{n} \leq 2 c_{2}^{s} W T \log n
\end{gathered}
$$

Inequality (12) is deduced by Chernoff bound and Inequality (13) is deduced by Hölder's Inequality. If the first term in Inequality (13) domains, we use Inequality (11).

$$
\begin{aligned}
\frac{\pi \Delta^{2} n\left(1+n^{\gamma}\right)}{4 m^{2} R^{4} \log ^{2} n} \frac{n \lambda^{s} n T}{c_{1}^{s} R^{2} \bar{D}^{s}} \mathbb{E}\left[r_{b}^{h}\right]^{2} \leq & 2 c_{2}^{s} W T \log n \\
\lambda^{s} \leq & \frac{8 c_{1}^{s} c_{2}^{s} W T}{\pi \Delta^{2}} \frac{m^{2} R^{6} \bar{D}^{s}}{n^{3}} \\
& \times \frac{\log ^{3} n}{\left(1+n^{\gamma}\right) \mathbb{E}\left[r_{b}^{h}\right]^{2}}
\end{aligned}
$$

The less $\mathbb{E}\left[r_{b}^{s}\right]$ and $\gamma$ are, the better tradeoff will be. Unfortunately, $\mathbb{E}\left[r_{b}^{s}\right]$ exist a minimum value $\Theta(\sqrt{m / n} R)$, because small $\mathbb{E}\left[r_{b}^{s}\right]$ may cause connectivity problem [1]. The Inequality (14) become

$$
\begin{aligned}
\lambda^{s} & \leq \frac{8 c_{1}^{s} c_{2}^{s} W T}{\pi \Delta^{2}} \frac{m R^{4} \bar{D}^{s} \log ^{3} n}{n^{2}} \\
\lambda^{s} & \leq O\left(\frac{m R^{4} \bar{D}^{s}}{n^{2}} \log ^{3} n\right)
\end{aligned}
$$

If the second term in Inequality (13) domains,we find $\lambda^{s} \leq$ $o\left(\frac{m R^{4} \bar{D}^{s}}{n^{2}} \log ^{3} n\right)$. Then we obtain the result with Inequality (15)

Theorem 3.1: Under cluster sparse regime, let $\overline{D^{s}}$ denote the mean delay averaged over all bits and let $\lambda^{s}$ be the
TABLE III: The order of optimal values of the scheduling parameters under cluster sparse regime when $D_{I I I}^{s}<D_{I I}^{s}$.

\begin{tabular}{|l|l|}
\hline$R_{d b}^{s}:$ \# of Intra-cluster duplications & $\Theta\left(n^{2-v-4 \beta-d} / \log ^{3} n\right)$ \\
\hline$R_{c_{b}^{s}}^{s}:$ \# of Inter-cluster duplications & $\Theta\left(n^{1-d-2 \beta} / \log n\right)$ \\
\hline$l_{b}^{s}:$ Capture Range & $\Theta\left(\min \left\{R, n^{\frac{3-v-6 \beta-2 d}{2}} / \log n\right\}\right)$ \\
\hline$h_{b}^{s}:$ \# of Hops & $\Theta\left(\min \left\{n^{\frac{1-v}{2}}, n^{2-v-4 \beta-d} / \log n\right\}\right)$ \\
\hline$r_{b}^{h}:$ Transmission range of Each Hop & $\Theta\left(n^{\frac{v-1+2 \beta}{2}}\right)$ \\
\hline
\end{tabular}

throughput of each source-destination pair. The following upper bound holds,

$$
\begin{cases}\left(\lambda^{s}\right)^{3} \leq O\left(\frac{m \bar{D}^{s}}{n} \log ^{3} n\right) & D_{I I I}^{s} \geq D_{I I}^{s} \\ \lambda^{s} \leq O\left(\frac{m R^{4} \bar{D}^{s}}{n^{2}} \log ^{3} n\right) & D_{I I I}^{s}<D_{I I}^{s}\end{cases}
$$

where $\lambda^{s} \leq m R^{2} / n$ and $\overline{D^{s}} \geq n /\left(m R^{2}\right)$

Proof: Using Lemma 3.6, Lemma 3.7, the minimum value of $D_{I I}^{s}$, and the maximum per-node throughput derived in [13], we can obtain the Theorem directly.

\section{Detailed Upper Bound of the Cluster Sparse REGIME}

In this section, we will obtain the optimal values of scheduling parameters and a delay-throughput tradeoff with detailed separation.

\section{A. Optimal values of scheduling parameters}

We assume that the mean delay is $\Theta\left(n^{d}\right)$. In order to obtain the tight upper bound of tradeoff, Equalities in inequalities (2), (4), (5) and (8) should hold, and some constrains such as $l_{b}^{s}$ $\left(l_{b}^{s} \leq R\right)$ and $r_{b}^{h}\left(r_{b}^{h} \geq \sqrt{m / n} R\right)$ should be considered. By solving these equations with constraints, we can obtain the optimal values of scheduling parameters. As these process are trivial, we omit it for simplification and show Table II and Table III directly.

\section{B. Detailed tradeoff with optimal values}

In this section, we will obtain a detailed picture about the delay-throughput tradeoff with optimal values of scheduling parameters. As a blurry separation $\left(D_{I I I}^{s} \geq D_{I I}^{s}\right.$ and $D_{I I I}^{s}<$ $D_{I I}^{s}$ ) is used in Theorem 3.1, which isn't a direct expression, we can use the optimal values of scheduling parameters to decide the precise separation of our upper bound.

The scheduling parameters suffer some common constraints, $R_{c b}^{s} \leq m, R_{d b}^{s} \leq q, l_{b}^{s} \leq R$ and $h_{b}^{s} \geq 1$. Other constraints are different for two situations $D_{I I I}^{s} \geq D_{I I}^{s}$ and $D_{I I I}^{s}<D_{I I}^{s}$, so we will discuss them separately.

Case 1: When $D_{I I I}^{s} \geq D_{I I}^{s}$

We solve the common constraints with the optimal values of scheduling parameters in Table II omitting the logarithmic factor. Discarding the meaningless results, we obtain $d \geq 1-v-2 \beta$ and $d \leq 1-v$, which are equivalent to $\bar{D}^{s} \geq n /\left(m R^{2}\right)$ and $\overline{D^{s}} \leq n / m$. These two inequalities are the nature lower bound [13] and upper bound of $\bar{D}^{s}$ for our cluster spare regime. 
There exist two other constraints. One is $D_{I I I}^{s} \geq D_{I I}^{s}$ and the other is $h_{b}^{s} \geq n R_{c b}^{s} /\left(m R^{2}\right)$. The first one also comes into the nature bound $\bar{D}^{s} \geq n /\left(m R^{2}\right)$ and the second one comes into $d \geq 5 / 2-v-6 \beta$, which is one of our targets. So our tradeoff can be partly written as

$$
\left(\lambda^{s}\right)^{3} \leq O\left(\frac{m \bar{D}^{s}}{n} \log ^{3} n\right) \quad d \geq \frac{5}{2}-v-6 \beta
$$

Case 2: when $D_{I I I}^{s}<D_{I I}^{s}$

We omit the solution of common constraints, which becomes the nature bound of our network. Another constraint is $D_{I I I}^{s}<D_{I I}^{s}$, which comes into $d<5 / 2-v-6 \beta$. Hence

$$
\lambda^{s} \leq O\left(\frac{m R^{2} \bar{D}^{s}}{n^{2}} \log ^{3} n\right) \quad d<\frac{5}{2}-v-6 \beta
$$

Theorem 4.1: Under cluster sparse regime, let $\bar{D}^{s}$ denote the mean delay averaged over all bits and let $\lambda^{s}$ be the throughput of each source-destination pair. The following upper bound holds,

$$
\begin{cases}\left(\lambda^{s}\right)^{3} \leq O\left(\frac{m \overline{D^{s}}}{n} \log ^{3} n\right) & d \geq \frac{5}{2}-v-6 \beta \\ \lambda^{s} \leq O\left(\frac{m R^{4} \bar{D}^{s}}{n^{2}} \log ^{3} n\right) & d<\frac{5}{2}-v-6 \beta\end{cases}
$$

where $\lambda^{s} \leq m R^{2} / n$ and $\overline{D^{s}} \geq n /\left(m R^{2}\right)$

Proof: Using Inequality (16), Inequality (17), the minimum value of $D_{I I}^{s}$, and the maximum per-node throughput derived in [13], we can obtain this theorem directly

\section{Lower Bound of the Cluster Sparse Regime}

We have gotten the upper bound as well as the optimal values of scheduling parameters, so we will construct an achievable lower bound in this section.

We divide our normal time slot into three subslots. The operations of each slot are shown below:

1) The nodes (source node and relays) create inter-cluster duplications and the destination cluster $C_{d}$ receives messages from inter-cluster duplications via one hop unicast with transmission range $r_{b}^{h}$.

2) $R_{d b}^{s}$ intra-cluster duplications are created via broadcast.

3) Intra-cluster duplication is captured by range $l_{b}^{s}$ and transmitted to the destination via $h_{b}^{s}$-hop unicast with single-hop transmission range $r_{b}^{h}$.

The scheduling parameters in our scheme use the optimal values in Table II and Table III. The operations in each slot are similar to the scheduling policy in our upper bound.

In each subslot, we tessellate the network into several cells and employ a cellular time-division multi-access (TDMA) transmission scheme so that each cell is scheduled to be active regularly. When a cell is activated, nodes within it are allowed to transmit to nodes inside the same cell or neighbouring cells. The TDMA transmission scheme allow each cell to have a $1 / c_{3}^{s}$ fraction of subslot to transmit, where $c_{3}^{s}$ is a constant being independent of the tessellation information. We describe our tradeoff achieving scheme and the network tessellation then.
1) In the 1st subslot, we divide each cluster $\Theta\left(R^{2}\right)$ into $\mathbb{T}_{1}^{s}=q=n^{1-v}$ equal-area cells. Assume that each message has a length of $\lambda^{s} / \log ^{2} n \leq m R^{2} /\left(n R_{c b}^{s}\right)$, and all transmissions are employed by one-hop unicast. So each node can transmit at least $n R_{c b}^{s} /\left(m R^{2}\right)$ messages when it is scheduled to be active. Each cluster has at least a chance of $\Theta\left(m R^{2} /(n \log n)\right)$ per time slot to communicate with other clusters, which indicates at least $R_{c b}^{s} / \log n$ messages can be sent per slot and network can sustain $\lambda^{s} / \log ^{2} n$ per slot throughput. If each time the network cannot sustain $\Theta\left(m R^{2} /(n \log n)\right)$ per-node throughput of inter-cluster communication, we denote it as Error $_{I}^{s}$. If the network falls to forward a message to its $C_{d}$ during $\Theta\left(D_{I I}^{s} \log ^{2} n\right)$ time slots, we denote it as rrror $_{I I}^{s}$.

2) \& 3) In the 2rd and 3th subslot, all messages are transmitted in their $C_{d}$. Nodes in a certain cluster follow the uniform distribution. The achievable lower bound under uniform condition have been studied widely that the network can achieve $\Theta\left(\lambda^{s} / \log n\right)$ throughput with $\Theta\left(\bar{D}^{s}\right)$ delay. But there exists a problem. If different clusters overlap at a certain area, they will take turns to transmit (under all three subslots). Error ${ }_{I I I}^{s}$ denote that more that $c_{4}^{s}$ overlap at a certain area, where $c_{4}^{s}$ is a positive number. So each cluster will take at least $1 / c_{4}^{s}$ length of subslot to transmit.

Theorem 5.1: Error $_{I}^{s} \rightarrow 0$, Error ${ }_{I I}^{s} \rightarrow 0$, and Error $_{I I I}^{s} \rightarrow 0$ as $n \rightarrow \infty$, So our lower bound under cluster sparse regime can achieve the per-node throughput of $\Theta\left(\lambda^{s} / \log ^{2} n\right)$ with $\Theta\left(\bar{D}^{s} \log ^{2} n\right)$ delay.

Proof: We start to prove that Error ${ }_{I}^{s} \rightarrow 0$, Error $_{I I}^{s} \rightarrow 0$, and Error $_{I I I}^{s} \rightarrow 0$ as $n \rightarrow \infty$. All the values of scheduling parameters used in the following proof are chosen from Table II and III.

1) Error $_{I}^{s}$ :

Let $\Lambda_{i}\left(i=1,2, \ldots, n^{2} /\left(m R^{2}\right)\right)$ be the amount of data can be transmit under a cell with area of $m R^{2} / n$ in the network. And $\Lambda=\sum_{i=1}^{n^{2} /\left(m R^{2}\right)} \Lambda_{i}$. The probability that at least two nodes from different clusters staying in the same cell area, which is first proposed in [13]:

$$
\begin{aligned}
E\left[\Lambda_{i}\right] \leq & \left(1-\left(1-\frac{(R-r / \sqrt{2})^{2}}{n}\right)^{m}\right)\left(1-\left(1-\frac{(R-r)^{2}}{n}\right)^{m-1}\right) \\
& \times\left(1-\left(1-\frac{r^{2}}{R^{2}}\right)^{q}\right)^{2}=\Theta\left(\frac{m^{2} R^{4}}{n^{2}}\right)
\end{aligned}
$$

By Chernoff bound, we can obtain that:

$$
\mathbf{P}\left[\Lambda<\Theta\left(\frac{m R^{2}}{\log n}\right)\right] \leq \frac{1}{e^{m R^{2} / 4}}<O\left(\frac{1}{n}\right)
$$

With $n \rightarrow \infty, \mathbf{P}\left[\Lambda<\Theta\left(m R^{2} / \log n\right)\right] \rightarrow 0$, which means that our network can at least sustain a pernode throughput of $\Theta\left(m R^{2} /(n \log n)\right)$ of inter-cluster communication. So $\mathbf{P}\left[\right.$ Error $\left._{I}^{s}\right] \rightarrow 0$, as $n \rightarrow \infty$

2) Error $_{I I}^{s}$ :

Equivalently, we can calculate the experiment that we throw $n \bar{D}^{s} \log ^{2} n$ balls to $\left(n / R_{c b}^{s}\right)\left(n / R^{2}\right)$ urns. If no ball falls in a certain urn, Error ${ }_{I I}^{s}$ happens (all value of the scheduling parameters are chosen from Table II and 
III). We denote the number of ball in each certain urn as $X_{d}^{s}$.

$$
\mathbb{E}\left[X_{d}^{s}\right]=\frac{n \bar{D}^{s} \log ^{2}}{\frac{n \bar{D}^{s} R^{2} \log n}{n} \frac{n}{R^{2}}}=\log n
$$

Using multiplicative form of Chernoff bound,

$$
\mathbf{P}\left[X_{d}^{s}=0\right]<\mathbf{P}\left[X_{d}^{s}<\frac{\log n}{2}\right]<\left(\sqrt{\frac{2}{e}}\right)^{\log n}=O\left(\frac{1}{n}\right)
$$

With $n \rightarrow \infty, \mathbf{P}\left[X_{d}^{s}=0\right] \rightarrow 0$, which indicates that $\mathbf{P}\left[\right.$ Error $\left._{I I}^{s}\right] \rightarrow 0$, as $n \rightarrow \infty$.

3) Error $_{I I I}^{s}$ :

From Fig 1, we know that if two cluster have a overlap part, their cluster center must stay in an circle with radius $R$. Using Chernoff's bound, let $X^{o}=\sum_{i=1}^{m} X_{i}^{o}$ be a random variable, with parameter $m$ and $R^{2} / n$ (the probability of success of each $X_{i}^{o}$ ).

$$
\mathbf{P}\left[X^{o}>c_{4}^{s}\right]<\left(\frac{m R^{2} e}{n}\right)^{\frac{n}{m R^{2}}}<O\left(\frac{1}{n}\right)
$$

With $n \rightarrow \infty, \mathbf{P}\left[X^{o}>c_{4}^{s}\right] \rightarrow 0$. The overlap in cluster sparse regime only affects the tradeoff with a constant factor, which indicates that $\mathbf{P}\left[\operatorname{Error}_{I I I}^{s}\right] \rightarrow 0$, as $n \rightarrow \infty$.

$\mathbf{P}\left[\right.$ Error $\left._{I}^{s}\right], \mathbf{P}\left[\right.$ Error $\left._{I I}^{s}\right]$, and $\mathbf{P}\left[\right.$ Error $\left._{I I I}^{s}\right]$ all come to 0 , as $n \rightarrow \infty$.

\section{Upper Bound of the Cluster Dense Regime}

Cluster dense regime (i.e., $v+2 \beta>1$ with weak node correlation) shows different properties from cluster sparse regime. The clusters here have a high probability to overlap. [19] tells us that every point in the network is covered by $\Theta\left(m R^{2} / n\right)=\Theta\left(n^{v+2 \beta-1}\right)$ clusters w.h.p, which indicates that nodes are almost distributed uniformly over the network domain. So the mean distance between two closet nodes is $\Theta(1)$. But it isn't truly uniform distribution. The correlated mobility model show some special phenomena which improve the delay-throughput tradeoff.

\section{A. Scheduling policy A}

In this section, we will first show some special phenomena under cluster dense regime. Then we will use these phenomena to design our scheduling policy A.

The phenomenon that every point in the network is covered by $\Theta\left(m R^{2} / n\right)=\Theta\left(n^{v+2 \beta-1}\right)$ clusters w.h.p seems helpful for us to create inter-cluster duplications under cluster dense regime, however it is not.

Lemma 6.1: Under cluster dense regime, an area of $\Theta\left(R^{2}\right)$ is covered by $\Theta\left(m R^{2} / n\right)$ clusters.

Proof: We assume $X_{i}^{o c}$ denote the event that a cluster and a certain area $\Theta\left(R^{2}\right)$ in the network overlap. And $X^{o c}=$ $\sum_{i=1}^{m} X_{i}^{o c}$. From Fig. 1, we can obtain

$$
\begin{aligned}
\mathbf{P}\left[X_{i}^{o c}=1\right] & =\frac{(2 R+r)^{2}}{n} \leq \frac{9 R^{2}}{n} \\
& =\frac{(2 R+r)^{2}}{n} \geq \frac{4 R^{2}}{n}
\end{aligned}
$$

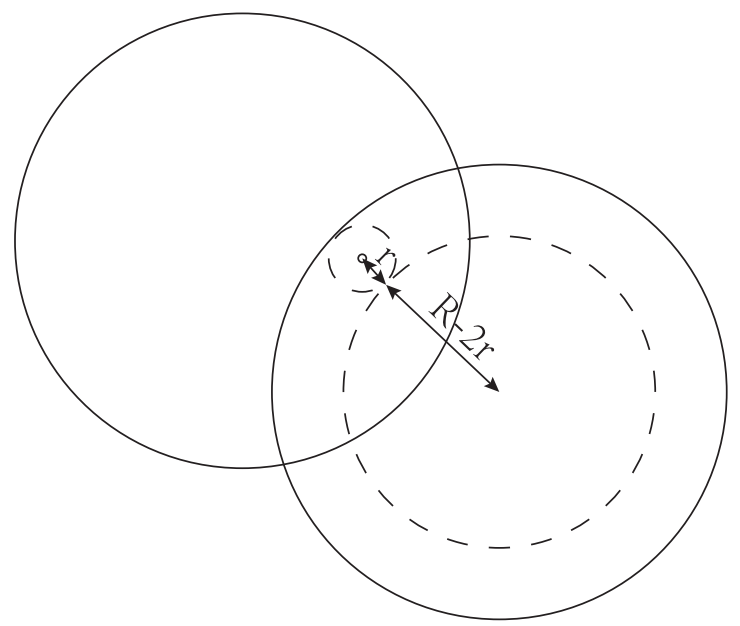

Fig. 3: Upper bound of inter-cluster transmission when only one node in source cluster contains message.

Using the multiplicative form of chernoff bound, we have

$$
\begin{aligned}
& \mathbf{P}\left[X_{\text {min }}^{o c}\right]=\mathbf{P}\left[X^{o c}>\frac{18 m R^{2}}{n}\right]<\left(\frac{e}{4}\right)^{\frac{9 m R^{2}}{n}}<O\left(\frac{1}{n}\right) \\
& \mathbf{P}\left[X_{\text {max }}^{o c}\right]=\mathbf{P}\left[X^{o c}<\frac{2 m R^{2}}{n}\right]<e^{-\frac{m R^{2}}{2 n}}<O\left(\frac{1}{n}\right)
\end{aligned}
$$

Since $0 \leq X^{o c} \leq m$, we have

$$
\begin{aligned}
& \mathbb{E}\left[X^{o c}\right]=\mathbb{E}\left[X^{o c} \mathbb{I}_{\left\{X_{m i n}^{\overline{o c}}\right\}}\right]+\mathbb{E}\left[X^{o c} \mathbb{I}_{\left\{X_{m i n}^{o c}\right\}}\right] \\
& \leq \frac{18 m R^{2}}{n}+m \frac{1}{n} \leq \frac{19 m R^{2}}{n} \\
& \mathbb{E}\left[X^{o c}\right]=\mathbb{E}\left[X^{o c} \mathbb{I}_{\left\{X_{\text {max }}^{\overline{o c}}\right\}}\right]+\mathbb{E}\left[X^{o c} \mathbb{I}_{\left\{X_{\text {max }}^{o c}\right\}}\right] \\
& \geq \frac{m R^{2}}{n}+0 \frac{1}{n}=\frac{m R^{2}}{n}
\end{aligned}
$$

Then

$$
\frac{m R^{2}}{n} \leq \mathbb{E}\left[X^{o c}\right] \leq \frac{19 m R^{2}}{n}
$$

So an area of $\Theta\left(R^{2}\right)$ is covered by $\Theta\left(m R^{2} / n\right)$ clusters.

Lemma 6.2: The probability that a source cluster send a message to a certain cluster successfully will not vary beyond a positive constant when the number of nodes containing the message in the source cluster changes. Assume transmitting range $r=o(R)$.

Proof: Fig.1 shows the best situation where all nodes in the source cluster contain the message. Then probability become $\Theta\left((2 R+r)^{2} / n\right)=\Theta\left(R^{2} / n\right)$

Fig. 3 shows the worst situation where only one node in the source cluster contains the message. Then probability become $\Theta\left((R-r)^{2} / n\right)=\Theta\left(R^{2} / n\right)$

The two extreme cases show us the lemma directly

These two properties discourage us from creating intercluster duplications with traditional broadcast or one-hop unicast manner. The latter method fails to utilize the cluster overlap under cluster dense regime, and the former method experience bad performs when creating more than $m R^{2} / n$ inter-cluster duplications. 
So we apply $u$ times broadcast with broadcast area $A_{d} \in$ $\left[1, \Theta\left(m R^{2} / n\right)\right]$. This operation performs well under cluster dense regime.

Lemma 6.3: If we have already created $R_{x} \leq \Theta(m)$ intercluster duplications, each point will still be covered with at least $\Theta\left(m R^{2} / n\right)$ clusters not containing a relay.

Proof: Assume $X^{e c}=\sum_{i=1}^{m R^{2} / n} X_{i}^{e c}$ is the number of clusters not containing duplications cover a certain point in the network. As there exists still $m-R_{x}=\Theta(m)$ cluster not containing duplication, $\operatorname{Pr}\left[X_{i}^{e c}\right]=\Theta(1)$. With chernoff bound,

$$
\operatorname{Pr}\left[X_{m i n}^{e c}\right]=\operatorname{Pr}\left[X^{e c}<\Theta\left(\frac{m R^{2}}{n}\right)\right]<e^{-\frac{m R^{2}}{8 n}}<O\left(\frac{1}{n}\right)
$$

Since $0 \leq X^{e c} \leq m$, we have

$$
\begin{aligned}
\mathbb{E}\left[X^{e c}\right] & =\mathbb{E}\left[X^{e c} \mathbb{I}_{\left\{X_{m i n}^{\overline{e c}}\right\}}\right]+\mathbb{E}\left[X^{o c} \mathbb{I}_{\left\{X_{m i n}^{e c}\right\}}\right] \\
& \geq \Theta\left(\frac{m R^{2}}{n}\right)+0 \frac{1}{n}=\Theta\left(\frac{m R^{2}}{n}\right)
\end{aligned}
$$

So each point will still be covered by at least $\Theta\left(m R^{2} / n\right)$ clusters not containing a relay.

At most $\Theta(m)$ inter-cluster duplications will be created, so $u$ times broadcast will be effectively operated. Each time when broadcasting, relays and source node can cooperate to create duplications; we can create $\Theta\left(A_{d}{ }^{u}\right)$ inter-cluster duplications during $u$ times broadcast, where $A_{d}$ is the broadcast area.

Now we introduce the scheduling policy A under cluster dense regime, and we illustrate the scheduling policy in Fig. 4. Opportunistic broadcast scheme is applied here.

1) Nodes containing a certain message create relays via $k$ th broadcast with area $A_{d}\left(k=1, \ldots, \Theta(u) ; R_{c}{ }^{d}\right.$ denotes the overall number of inter-cluster duplications).

2) If one relay is captured by a node in $C_{d}$ with range $l_{1}{ }^{d}$, the message will be transmitted to the node via $h_{1}{ }^{d}$-hop unicast. If not, we come back to step 1).

3) Relays in $C_{d}$ create new intra-cluster duplications via broadcast $\left(R_{d}{ }^{d}\right.$ denotes the overall number of intracluster duplications).

4) If one intra-cluster duplication is captured by its destination with range $l_{2}{ }^{d}$, the message will be transmitted to the destination via $h_{2}{ }^{d}$-hop unicast.If not, we come back to step 3).

But the scheduling policy A only performs well when the node correlation is relatively high. Another schedule will be designed and discussed in section $\mathrm{VI}-\mathrm{H}$, when nodes show weak correlations. In the following analysis, we divide our scheduling policy A into two parts. One is 1)-2) (Part I) and the other is 3)-4) (Part II). We will analyse them respectively.

\section{B. Tradeoff for delay of Part I}

In this section, we first will give an intuitive explanation ; then we give the analysis for sophisticated strategy shown above.

We denote $D_{I 1}^{d}$ as the delay of creating $R_{c}{ }^{d}$ inter-cluster duplications, and denote $D_{I I 1}^{d}$ as the delay of forwarding a message from $R_{c}{ }^{d}$ inter-cluster duplications to a node in $C_{d}$.
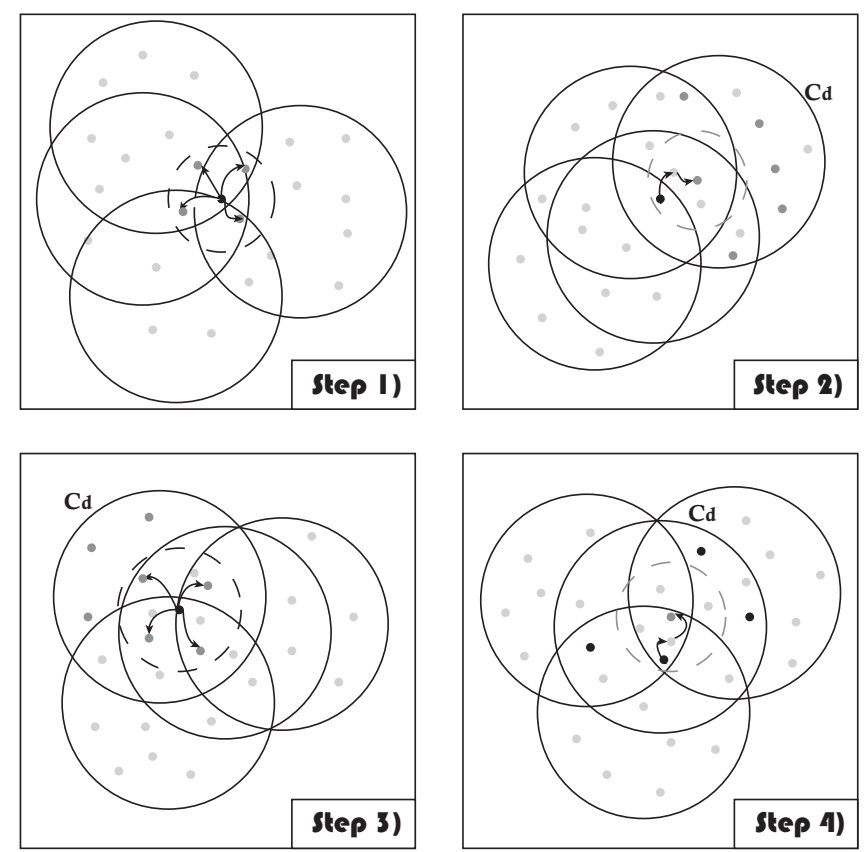

Fig. 4: Scheduling policy A under cluster dense regime.

$D_{I 1}^{d}$ is the delay for $u$ times broadcast. $R_{c}{ }^{s}=A_{d}{ }^{u}$ will be created within $u$ times $\left(A_{d} \in\left[1, \Theta\left(m R^{2} / n\right)\right]\right)$. When $R_{c}{ }^{d}=$ $\omega(1)=n^{\hat{a}}$ and $A_{d}=n^{\alpha}$, where $\hat{a}$ and $\alpha$ are two constants greater than 0 . Hence

$$
u=\frac{\log n^{\hat{a}}}{\log n^{\alpha}}=\frac{\hat{a}}{\alpha}=\Theta(1)
$$

When $R_{c}{ }^{d}=\Theta(1)$, we let broadcast area $A_{d}=\Theta\left(R_{c}{ }^{d}\right)$. $D_{I 1}^{d}$ is still bounded by $\Theta(1)$, which indicates the $D_{I 1}^{d}$ is negligible. Even under the condition that $A_{d}=\Theta(1)$ (cluster critical regime), delay can still be bounded by $\Theta(\log n)$.

$D_{I I}^{d}$ is the delay for nodes in $C_{d}$ catch one of the intercluster duplications, so

$$
\begin{aligned}
D_{I I 1}^{d} & =\frac{1}{\left(1-\left(1-\frac{R^{2}}{n}\right) R_{c}{ }^{d}\right) \frac{l_{1}^{d^{2} n / m}}{R^{2}}} \\
& \geq \frac{m}{R_{c}{ }^{d} l_{1}^{d^{2}}}
\end{aligned}
$$

Even we use the "opportunistic duplication scheme", delay can only be improved with a $\log n$ factor.

Lemma 6.4: Under the cluster dense regime and scheduling policy A, the delay for a particular bit $b$ of Part I and its scheduling parameters comply the following inequality

$$
c_{1}^{d} \log n \mathbb{E}\left[D_{b 1}^{d}\right] \geq \frac{m}{\mathbb{E}\left[R_{c b}^{d}\right] \mathbb{E}\left[h_{1 b}^{d}+\frac{1}{n}\right]^{2}}
$$

where $c_{1}^{d}$ is a positive constant and variable $X_{b}^{d}$ denote the variable $X$ under cluster dense regime for a particular bit $b$.

The proof of Lemma 6.4 is similar to the proof in Appendix A, so we omit it for simplification. 


\section{Tradeoff for Radio Resource, Half Duplex and Mutihop of} Part I

The radio resource of $u$ times broadcast is similar to traditional broadcast. So we can obtain the tradeoff for radio resource with opportunistic broadcast scheme.

Lemma 6.5: Under cluster dense regime, scheduling policy $\mathrm{A}$, and concerning radio resource, the following inequality holds

$\sum_{b=1}^{\lambda_{1}^{d} n T} \frac{\Delta^{2}}{4} \frac{\mathbb{E}\left[R_{c b}^{d}\right]-1}{n}+\mathbb{E}\left[\sum_{b=1}^{\lambda_{1}^{d} n T} \sum_{h=1}^{h_{1}^{d}} \frac{\pi \Delta^{2}}{4} \frac{r_{b}^{h^{2}}}{n}\right] \leq c_{2}^{d} W T \log n$

where $c_{2}^{d}$ is a positive number, $h_{1 b}^{d}$ is the number of transmission hops after message being captured by a node in $C_{d}$, and $r_{b}^{h}$ is the transmission range of each hop.

Since no node can transmit and receive at the same time and over same frequency, the following inequality holds,

Lemma 6.6: The following inequality holds,

$$
\sum_{b=1}^{\lambda_{1}^{d} n T} \sum_{h=1}^{h_{1}^{d}} 1 \leq \frac{W T}{2} n
$$

The following inequality holds for the nature of multihop.

Lemma 6.7: The following inequality holds,

$$
\sum_{b=1}^{\lambda_{1}^{d} n T} \sum_{h=1}^{h_{1}^{d}} r_{b}^{h} \geq l_{1 b}^{d}
$$

\section{Detailed upper bound on delay-throughput tradeoff of Part}

I

In this section, we first derive one part of the delaythroughput tradeoff on the basis of fundamental tradeoffs. Then we achieve the optimal values of scheduling parameters. Finally we obtain the other part of tradeoff by the optimal values and constraints of the scheduling parameters.

From Lemma 6.4, we have

$$
\begin{aligned}
\sum_{b=1}^{\lambda_{1}^{d} n T} \mathbb{E}\left[R_{c b}{ }^{d}\right] \geq & \frac{1}{c_{1}^{d} \log n} \sum_{b=1}^{\lambda_{1}^{d} n T} \frac{m}{\left(\mathbb{E}\left[l_{1 b}^{s}\right]+\frac{1}{n}\right)^{2} \mathbb{E}\left[D_{1 b}^{d}\right]} \\
\geq & \frac{m}{c_{1}^{d} \log n} \frac{\sum_{b=1}^{\lambda_{1}^{d} n T} 1}{\sum_{b=1}^{\lambda_{b}^{d} n T} \mathbb{E}\left[D_{1 b}^{d}\right]} \\
& \times \frac{\left(\sum_{b=1}^{\lambda_{1}^{d} n T} 1\right)^{3}}{\left(\sum_{b=1}^{\lambda_{1}^{d} n T}\left(\mathbb{E}\left[l_{1 b}^{d}\right]+\frac{1}{n}\right)\right)^{2}} \\
= & \frac{m}{c_{1}^{d} \log n} \frac{\left(\sum_{b=1}^{\lambda_{1}^{d} n T} 1\right)^{3}}{\bar{D}_{1}^{s}\left(\sum_{b=1}^{\lambda_{1}^{s} n T}\left(\mathbb{E}\left[l_{1}^{s}\right]+\frac{1}{n}\right)\right)^{2}}
\end{aligned}
$$

Inequality (22) is deduced by using Jensen's Inequality and Hölder's Inequality. From Lemma 6.5 and Cauchy-Schwartz inequality, we obtain

$$
\begin{aligned}
& \sum_{b=1}^{\lambda_{1}^{d} n T} \frac{\Delta^{2}}{4} \frac{\mathbb{E}\left[R_{c b}^{d}\right]-1}{n}+\frac{\pi \Delta^{2}}{2 W T n^{2}}\left(\sum_{b=1}^{\lambda_{1}^{d} n T} \mathbb{E}\left[l_{1 b}^{d}\right]\right)^{2} \leq c_{2}^{d} W T \log n \\
& \frac{\Delta^{2} m}{4 c_{1}^{d} n \log n} \frac{\left(\sum_{b=1}^{\lambda_{1}^{d} n T} 1\right)^{3}}{D_{1}^{d}\left(\sum_{b=1}^{\lambda_{1}^{d} n T}\left(\mathbb{E}\left[l_{1 b}^{d}\right]+\frac{1}{n}\right)\right)^{2}} \\
& +\frac{\pi \Delta^{2}}{2 W T n^{2}}\left(\sum_{b=1}^{\lambda_{1}^{d} n T} \mathbb{E}\left[l_{1 b}^{d}\right]\right)^{2}-\frac{\Delta^{2}}{4} \lambda^{d} T \leq c_{2}^{s} W T \log n \\
& \text { If } \sum_{b=1}^{\lambda_{1}^{d} n T}\left[l_{1 b}^{d}\right]<\lambda_{1}^{d} T, \\
& \frac{\Delta^{2} m}{4 c_{1}^{d} n \log n} \frac{\left(\lambda_{1}^{d} n T\right)^{3} n^{2}}{\bar{D}_{1}^{d}\left(\lambda_{1}^{d} T\right)^{2}} \leq c_{2}^{d} W T \log n \\
& \lambda_{1}^{d} \leq \frac{4 c_{1}^{d} c_{2}^{d} W T \overline{D_{1}^{d}} \log ^{2} n}{\Delta^{2} n^{5} m T}
\end{aligned}
$$

If $\sum_{b=1}^{\lambda_{1}^{d} n T}\left[l_{1 b}^{d}\right] \geq \lambda_{1}^{d} T$

$$
\begin{aligned}
& \frac{\Delta^{2} m}{4 c_{1}^{d} n \log n} \frac{\left(\sum_{b=1}^{\lambda_{1}^{d} n T} 1\right)^{3}}{\overline{D_{1}^{d}\left(\sum_{b=1}^{\lambda_{1}^{d} n T}\left(\mathbb{E}\left[l_{1 b}^{d}\right]\right)^{2}\right.}} \\
& +\frac{\pi \Delta^{2}}{2 W T n^{2}}\left(\sum_{b=1}^{\lambda_{1}^{d} n T} \mathbb{E}\left[l_{1}^{d}\right]\right)^{2} \leq c_{2}^{d} W T \log n \\
& \sqrt{\frac{\pi \Delta^{2} T^{2}}{8 c_{1}^{d} W \log n} \frac{\left(\lambda_{1}^{d}\right)^{3} m}{\bar{D}_{1}^{d}}} \leq c_{2}^{d} W T \log n
\end{aligned}
$$

$$
\left(\lambda_{1}^{d}\right)^{3} \leq \frac{8 c_{1}^{d} c_{2}^{d^{2}} W^{3} \bar{D}_{1}^{s} \log ^{3} n}{\pi \Delta^{2} m}
$$

Compare the two inequalities (23) and (25), hence

$$
\left(\lambda_{1}^{d}\right)^{3} \leq O\left(\frac{\overline{D_{1}^{d}}}{m} \log ^{3} n\right)
$$

But it isn't the final result for delay-throughput tradeoff of cluster dense regime. We assume that the mean delay is $\Theta\left(n^{d}\right)$. In order to obtain the tight upper bound of the tradeoff, equalities in inequalities (18), (20), (21) and (24) should hold. The optimal values of scheduling parameters are shown in Table IV.

There exists two constraints for our scheduling parameters $1 \leq R_{c b}^{d} \leq m$ and $1 \leq l_{1 b}^{d} \leq \sqrt{m R^{2} / n}$. By using optimal values in Table IV and omitting the logarithmic factor, we obtain $d \geq(3-v-6 \beta) / 2$. It means that $l_{1 b}^{d}=\sqrt{m R^{2} / n}$ should maintain, if we reach $d<(3-v-6 \beta) / 2$. Then Lemma 6.4 becomes

$$
c_{1}^{d} \log n \mathbb{E}\left[D_{b 1}^{s}\right] \geq \frac{n}{\mathbb{E}\left[R_{c b}^{d}\right] R^{2}}
$$

And Lemma 6.5 comes into 
TABLE IV: The order of optimal values of the scheduling parameters under Part I of cluster dense regime and scheduling policy A I.

\begin{tabular}{|l|l|}
\hline$R_{c_{b}}^{d}:$ \# of Inter-cluster duplications & $\Theta\left(n^{\frac{v-d}{3}} / \log n\right)$ \\
\hline$l_{1}^{d}:$ Capture Range & $\Theta\left(n^{\frac{v-d}{3}} / \log \frac{1}{2} n\right)$ \\
\hline$h_{1}^{d}:$ \# of Hops & $\Theta\left(n^{\frac{v-d}{3}} / \log n\right)$ \\
\hline$r_{b}^{h}:$ Transmission range of Each Hop & $\Theta\left(\log ^{\frac{1}{2}} n\right)$ \\
\hline
\end{tabular}

$$
\begin{aligned}
\sum_{b=1}^{\lambda_{1}^{d} n T} \frac{\Delta^{2}}{4} \frac{\mathbb{E}\left[R_{c b}^{d}\right]}{n}+\frac{\pi \Delta^{2}}{2 W T n^{2}}\left(\sum_{b=1}^{\lambda_{1}^{d} n T} \sqrt{\frac{m R^{2}}{n}}\right)^{2} & \leq c_{2}^{d} W T \log n \\
O\left(\frac{\lambda_{1}^{d} n}{R^{2} \bar{D}_{1}^{d} \log ^{2} n}+\frac{\left(\lambda_{1}^{d}\right)^{2} m R^{2}}{n \log ^{2} n}\right) & \leq \log n
\end{aligned}
$$

$$
\lambda_{1}^{d} \leq O\left(\frac{R^{2} \bar{D}_{1}^{d}}{n} \log ^{3} n\right) \sum_{b=1}^{\lambda_{2}^{d} n T} \frac{\Delta^{2}}{4} \frac{\frac{m R^{2}}{n}\left(\mathbb{E}\left[R_{d b}^{d}\right]-1\right)}{n}+\mathbb{E}\left[\sum_{b=1}^{\lambda_{2}^{d} n T} \sum_{h=1}^{h_{2}^{d}} \frac{\pi \Delta^{2}}{4} \frac{r_{b}^{h^{2}}}{n}\right] \leq c_{4}^{d} W T \log n
$$

Then we can calculate the optimal values of scheduling parameters for the other part of tradeoff $(d<(3-v-6 \beta) / 2)$, which is shown in Table $\mathrm{V}$.

Theorem 6.1: Under cluster dense regime and scheduling policy A, let $\bar{D}_{1}^{d}$ denote the mean delay averaged over all bits and let $\lambda_{1}^{d}$ be the throughput of each source-destination pair. In Part I, the following upper bound holds,

$$
\begin{cases}\left(\lambda_{1}^{d}\right)^{3} \leq O\left(\frac{\overline{D_{1}^{d}}}{m} \log ^{3} n\right) & d \geq \frac{3-v-6 \beta}{2} \\ \lambda_{1}^{d} \leq O\left(\frac{R^{2} \overline{D_{1}^{d}}}{n} \log ^{3} n\right) & d<\frac{3-v-6 \beta}{2}\end{cases}
$$

\section{E. Tradeoff for delay of Part II}

$D_{I 2}^{d}$ is the delay for $R_{d}^{d}$ intra-cluster duplications being captured by the destination with range $l_{2}^{d}$. We can obtain

$$
\begin{aligned}
D_{I 2}^{d} & =\frac{1}{\left(1-\left(1-\frac{l_{2}^{d 2}}{R^{2}}\right)^{R_{d}{ }^{d}}\right)} \\
& \geq \frac{R^{2}}{R_{d}{ }^{d} l_{2}^{d^{2}}}
\end{aligned}
$$

Even we use the opportunistic duplication scheme, delay can only be improved with a $\log n$ factor.

Lemma 6.8: Under the cluster dense regime and scheduling policy A, the delay for a particular bit $b$ of Part II and its scheduling parameters comply the following inequality

$$
c_{3}^{d} \log n \mathbb{E}\left[D_{b 2}^{d}\right] \geq \frac{R^{2}}{\mathbb{E}\left[R_{d b}^{d}\right] \mathbb{E}\left[l_{2 b}^{d}+\frac{1}{n}\right]^{2}}
$$

The proof of Lemma 6.8 is similar to the proof in Appendix A, so we omit it for simplification.

\section{F. Tradeoff for Radio Resource, Half Duplex and Mutihop of Part II}

Similar to Part I, the radio resource and some scheduling parameters comply the following tradeoff. Opportunistic broadcast scheme is applied here.
Lemma 6.9: Under cluster dense regime, scheduling policy $\mathrm{A}$, and concerning radio resource, the following inequality holds

TABLE V: The order of optimal values of the scheduling parameters under Part I of cluster dense regime and scheduling policy A II.

\begin{tabular}{|l|l|}
\hline$R_{c_{b}^{d}}:$ \# of Inter-cluster duplications & $\Theta\left(n^{1-2 \beta-d} / \log n\right)$ \\
\hline$l_{1}^{d}$ : Capture Range & $\Theta\left(n^{\frac{v+2 \beta-1}{2}} / \log ^{\frac{1}{2}} n\right)$ \\
\hline$h_{1}{ }_{b}^{d}:$ \# of Hops & $\Theta\left(n^{\frac{v+2 \beta-1}{2}} / \log n\right)$ \\
\hline$r_{b}^{h}:$ Transmission range of Each Hop & $\Theta\left(\log ^{\frac{1}{2}} n\right)$ \\
\hline
\end{tabular}

where $c_{4}^{d}$ is a positive number, $h_{2 b}^{s}$ is the number of transmission hops after message being captured by the destination, and $r_{b}^{h}$ is the transmission range of each hop.

Since no node can transmit and receive at the same time and over same frequency, the following inequality holds,

Lemma 6.10: The following inequality holds,

$$
\sum_{b=1}^{\lambda_{2}^{d} n T} \sum_{h=1}^{h_{2}^{d}} 1 \leq \frac{W T}{2} n
$$

The following inequality holds for the nature of multihop.

Lemma 6.11: The following inequality holds,

$$
\sum_{b=1}^{\lambda_{2}^{d} n T} \sum_{h=1}^{h_{2}^{d}} r_{b}^{h} \geq l_{2 b}^{d}
$$

\section{G. Detailed upper bound on delay-throughput tradeoff of Part II}

In this section, we first derive one part of the delaythroughput tradeoff on the basis of fundamental tradeoffs. Then we obtain the optimal values of scheduling parameters. Finally we obtain the other part of tradeoff by optimal values and constraints of the scheduling parameters.

From Lemma 6.8, we have

$$
\begin{aligned}
\sum_{b=1}^{\lambda_{2}^{d} n T} \mathbb{E}\left[R_{d b}^{d}\right] & \geq \frac{1}{c_{3}^{d} \log n} \sum_{b=1}^{\lambda_{2}^{d} n T} \frac{R^{2}}{\left(\mathbb{E}\left[l_{2}^{s}\right]+\frac{1}{n}\right)^{2} \mathbb{E}\left[D_{2 b}^{d}\right]} \\
& \geq \frac{R^{2}}{c_{3}^{d} \log n} \frac{\left(\sum_{b=1}^{\lambda_{2}^{d} n T} 1\right)^{3}}{\overline{D_{2}^{s}}\left(\sum_{b=1}^{\lambda_{2}^{s} n T}\left(\mathbb{E}\left[l_{2 b}^{s}\right]+\frac{1}{n}\right)\right)^{2}}
\end{aligned}
$$


TABLE VI: The order of the optimal values of the scheduling parameters under Part II of cluster dense regime and scheduling policy A I.

\begin{tabular}{|l|l|}
\hline$R_{d b}^{d}:$ \# of Inter-cluster duplications & $\Theta\left(n^{\frac{2-2 v-2 \beta-d}{3}} / \log n\right)$ \\
\hline$l_{2}{ }_{b}^{d}:$ Capture Range & $\Theta\left(n^{\frac{v+4 \beta-1-d}{3}} / \log \frac{1}{2} n\right)$ \\
\hline$h_{2}{ }_{b}:$ \# of Hops & $\Theta\left(n^{\frac{v+4 \beta-1-d}{3}} / \log n\right)$ \\
\hline$r_{b}^{h}:$ Transmission range of Each Hop & $\Theta\left(\log ^{\frac{1}{2}} n\right)$ \\
\hline
\end{tabular}

From Lemma 6.9, Cauchy-Schwartz inequality

$$
\begin{aligned}
& \sum_{b=1}^{\lambda_{2}^{d} n T} \frac{\Delta^{2}}{4} \frac{m R^{2} \mathbb{E}\left[R_{d b}^{d}\right]}{n^{2}}+\frac{\pi \Delta^{2}}{2 W T n^{2}}\left(\sum_{b=1}^{\lambda_{2}^{d} n T} \mathbb{E}\left[l_{2 b}^{d}\right]\right)^{2} \leq c_{4}^{d} W T \log n \\
& \frac{\Delta^{2} m R^{4}}{4 c_{3}^{d} n^{2} \log n} \frac{\left(\sum_{b=1}^{\lambda_{2}^{d} n T} 1\right)^{3}}{\overline{D_{2}^{d}\left(\sum_{b=1}^{\lambda_{2}^{d} n T}\left(\mathbb{E}\left[l_{2 b}^{d}\right]+\frac{1}{n}\right)\right)^{2}}} \\
& +\frac{\pi \Delta^{2}}{2 W T n^{2}}\left(\sum_{b=1}^{\lambda_{2}^{d} n T} \mathbb{E}\left[l_{2 b}^{d}\right]\right)^{2} \leq c_{4}^{s} W T \log n
\end{aligned}
$$

If $\sum_{b=1}^{\lambda_{2}^{d} n T}\left[l_{2 b}^{d}\right]<\lambda_{2}^{d} T$,

$$
\begin{aligned}
\frac{\Delta^{2} m R^{4}}{4 c_{3}^{d} n^{2} \log n} \frac{\left(\lambda_{2}^{d} n T\right)^{3} n^{2}}{\overline{D_{2}^{d}\left(\lambda^{d} T\right)^{2}}} & \leq c_{4}^{d} W T \log n \\
\lambda_{2}^{d} & \leq \frac{4 c_{3}^{d} c_{4}^{d} W T \bar{D}_{1}^{d} \log ^{2} n}{\Delta^{2} n^{4} m R^{2} T}
\end{aligned}
$$

If $\sum_{b=1}^{\lambda_{2}^{d} n T}\left[l_{2 b}^{d}\right] \geq \lambda_{2}^{d} T$,

$$
\begin{aligned}
\frac{\Delta^{2} m R^{4}}{4 c_{1}^{d} n^{2} \log n} \frac{\left(\sum_{b=1}^{\lambda_{2}^{d} n T} 1\right)^{3}}{\bar{D}_{2}^{d}\left(\sum_{b=1}^{\lambda_{2}^{d} n T}\left(\mathbb{E}\left[l_{2 b}^{d}\right]\right)^{2}\right.} & \\
+\frac{\pi \Delta^{2}}{2 W T n^{2}}\left(\sum_{b=1}^{\lambda_{2}^{d} n T} \mathbb{E}\left[l_{2 b}^{d}\right]\right)^{2} & \leq c_{4}^{d} W T \log n \\
\sqrt{\frac{\pi \Delta^{2} T^{2}}{8 c_{3}^{d} W \log n} \frac{\left(\lambda_{2}^{d}\right)^{3} m R^{4}}{n \bar{D}_{2}^{d}}} & \leq c_{4}^{d} W T \log n \\
\left(\lambda_{2}^{d}\right)^{3} & \leq \frac{8 c_{3}^{d} c_{4}^{d^{2}} W^{3} n \bar{D}_{2}^{s} \log ^{3} n}{\pi \Delta^{2} m R^{4}}
\end{aligned}
$$

Compare the two inequalities (30) and (32), hence

$$
\left(\lambda_{2}^{d}\right)^{3} \leq O\left(\frac{n \bar{D}_{2}^{d}}{m R^{4}} \log ^{3} n\right)
$$

But it isn't the final result for delay-throughput tradeoff. We assume that the mean delay is $\Theta\left(n^{d}\right)$. In order to obtain the tight upper bound of the tradeoff, inequities in inequalities (26), (28), (29) and (31) should hold. The optimal values of scheduling parameters are shown in Table VI.

There exists a constraint for our scheduling parameters $1 \leq$ $R_{d b}^{d} \leq n / m$. By using optimal values in Table VI and omitting the logarithmic factor, we obtain $d \leq 2-2 v-2 \beta$. It means
TABLE VII: The order of the optimal values of the scheduling parameters under Part II of cluster dense regime and scheduling policy A II.

\begin{tabular}{|l|l|}
\hline$R_{d b}^{d}:$ \# of Inter-cluster duplications & $\Theta(1)$ \\
\hline$l_{2}^{d}:$ Capture Range & $\Theta\left(n^{\frac{2 \beta-d}{2}} / \log ^{\frac{1}{2}} n\right)$ \\
\hline$h_{2}^{d}:$ \# of Hops & $\Theta\left(n^{\frac{2 \beta-d}{2}} / \log n\right)$ \\
\hline$r_{b}^{h}:$ Transmission range of Each Hop & $\Theta\left(\log ^{\frac{1}{2}} n\right)$ \\
\hline
\end{tabular}

that $R_{d b}^{d}=1$ should maintain, if we reach $d>2-2 v-2 \beta$. Then Lemma 6.8 become

$$
c_{3}^{d} \log n \mathbb{E}\left[D_{b 2}^{s}\right] \geq \frac{R^{2}}{\mathbb{E}\left[l_{2 b}^{d}\right]^{2}}
$$

And Lemma 6.9 comes into

$$
\begin{aligned}
\sum_{b=1}^{\lambda_{2}^{d} n T} \frac{\Delta^{2}}{4} \frac{m R^{2}}{n^{2}}(1-1)+\frac{\pi \Delta^{2}}{2 W T n^{2}} \mathbb{E}\left[\sum_{b=1}^{\lambda_{2}^{d} n T} l_{2 b}^{h}\right]^{2} & \leq c_{4}^{d} W T \log n \\
\frac{\pi \Delta^{2}}{4 W T n^{2}} \frac{\mathbb{E}\left[l_{2 b}^{d}\right]^{2}\left(\lambda_{2}^{d} n T\right)^{2}}{\log n} & \leq c_{4}^{d} W T \log n \\
\left(\lambda_{2}^{d}\right)^{2} & \leq O\left(\frac{\bar{D}_{2}^{d}}{R^{2}} \log ^{3} n\right)
\end{aligned}
$$

Then we can calculate the optimal values of scheduling parameters for the other part of tradeoff $(d \geq 2-2 v-2 \beta)$, which is shown in Table VII.

Theorem 6.2: Under cluster dense regime and scheduling policy A, let $\bar{D}_{2}^{d}$ denote the mean delay averaged over all bits and let $\lambda_{2}^{d}$ be the throughput of each source-destination pair. In Part II, the following upper bound holds,

$$
\begin{cases}\left(\lambda_{2}^{d}\right)^{2} \leq O\left(\frac{\bar{D}_{2}^{d}}{R^{2}} \log ^{3} n\right) & d \geq 2-2 v-2 \beta \\ \left(\lambda_{2}^{d}\right)^{3} \leq O\left(\frac{n \bar{D}_{1}^{d}}{m R^{4}} \log ^{3} n\right) & d<2-2 v-2 \beta\end{cases}
$$

H. Scheduling Policy B and Overall Upper bound of delaythroughput tradeoff

Theorem 6.1 and 6.2 show us the tradeoff of Part I and Part II of scheduling policy A respectively. We assume $\bar{D}_{1}^{d}=$ $\bar{D}_{2}^{d}=\bar{D}_{A}^{d}$. Then the upper bound of scheduling policy A can be derived easily.

Theorem 6.3: Under cluster dense regime and scheduling policy A, let $D_{A}^{d}$ denote the mean delay averaged over all bits and let $\lambda_{A}^{d}$ be the throughput of each source-destination pair. Assume $D_{1}^{d}=\bar{D}_{2}^{d}=\bar{D}_{A}^{d}$. The following upper bound holds,

$$
\lambda_{A}^{d}=\min \left\{\lambda_{1}^{d}, \lambda_{2}^{d}\right\}
$$

But this is not the end of story, our scheduling policy A will become inefficient when nodes show extremely weak correlation (i.e., too many clusters or each cluster covers a large area). Scheduling policy A is base on the principle that the transmission within a certain cluster $\left(C_{d}\right)$ can save time and radio resource, however nodes within the same cluster will occasional meet each other and broadcast operation of creating intra-cluster duplications needs more radio resource, when the 

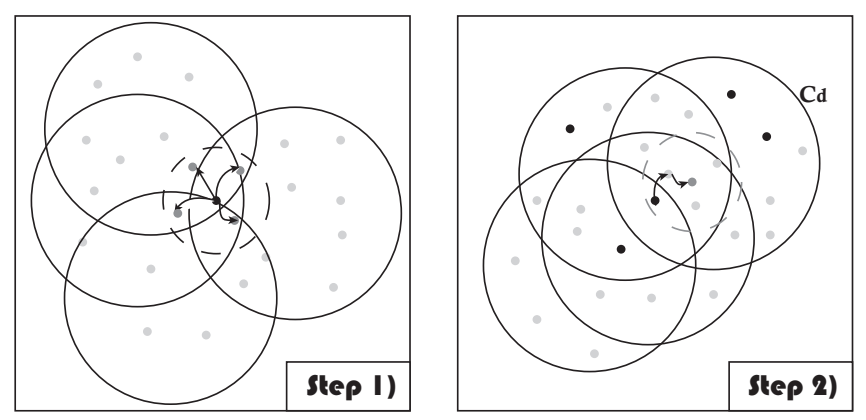

Fig. 5: Scheduling policy B under cluster dense regime.

nodes' correlation are weak. So we propose the scheduling policy B, and we illustrate the policy in Fig. 5. Opportunistic broadcast scheme is applied here.

1) Nodes containing a certain message create relays via $k$ th broadcast with area $A_{d}\left(k=1, \ldots, \Theta(u) ; R_{b}^{d}\right.$ denotes the overall relays).

2) If one relay is captured by the destination with range $l_{b}^{d}$, the message will be transmitted to the destination via $h_{b}^{d}$-hop unicast. If not, we come back to step 1).

In order to obtain the delay-throughput tradeoff upper bound, we have to solve the basic tradeoff firstly. Fortunately, the basic tradeoff of radio resource, multihop, and half duplex is same to the i.i.d. model. So we only need to solve the basic tradeoff of delay.

Lemma 6.12: Under the cluster dense regime and scheduling policy $\mathrm{B}$, the delay for a particular bit $b$ and its scheduling parameters comply the following inequality

$$
c_{6}^{d} \log n \mathbb{E}\left[D_{b}^{d}\right] \geq \frac{n}{\mathbb{E}\left[R_{b}^{d}\right] \mathbb{E}\left[l_{b}^{d}+\frac{1}{n}\right]^{2}}
$$

where $c_{6}^{d}$ is a positive constant.

Proof: Here we only give the intuitive proof. The detailed proof is shown in Appendix B

We assume the broadcast create $R_{b i}^{d}$ relays in $\hat{m}$ cluster, where $i=1,2, \ldots, \hat{m}$. We denote $X^{c a p}$ as the event that at least one relay is capture by the destination, then

when $l_{b}^{d} \leq \Theta(R)$ :

$$
\begin{aligned}
\mathbf{P}\left[X^{c a p}\right] & \leq 1-\prod_{i=1}^{\hat{m}}\left(1-\frac{\left(R-l_{b}^{d}\right)^{2}}{n} \frac{\left(l_{b}^{d}\right)^{2}}{R^{2}}\right)^{R_{b i}^{d}} \\
& \leq 1-\left(1-\frac{c_{6}^{d}\left(l_{b}^{d}\right)^{2}}{n}\right)^{R_{b}^{d}} \leq \frac{c_{6}^{d}\left(l_{b}^{d}\right)^{2} R_{b}^{d}}{n}
\end{aligned}
$$

when $l_{b}^{d}>\Theta(R)$ : we treat relays as $\Theta\left(R_{b}^{d}\right)$ inter-cluster duplications, because they are created by $u$ times broadcast.

$$
\mathbf{P}\left[X^{c a p}\right] \leq 1-\left(1-\frac{c_{6}^{d}\left(l_{b}^{d}\right)^{2}}{n}\right)^{R_{b}^{d}} \leq \frac{c_{6}^{d}\left(l_{b}^{d}\right)^{2} R_{b}^{d}}{n}
$$

Now, it is easy to show that the average packet delay needed is:

$$
D_{b}^{d}=\frac{1}{\mathbf{P}\left[X^{c a p}\right]} \geq \frac{n}{c_{6}^{d} R_{b}^{d}\left(l_{b}^{d}\right)^{2}}
$$

By Hölder's Inequality and Jensen's Inequality:

$$
c_{6}^{d} \mathbb{E}\left[D_{b}^{d}\right] \geq \frac{n}{\mathbb{E}\left[R_{b}^{d}\right] \mathbb{E}\left[l_{b}^{d}\right]^{2}}
$$

If the relays needed is larger than the cluster number $m$ when $l_{b}^{d}>\Theta(R)$, this basic tradeoff will fall. But it won't happen, which will be shown in section VII-B.

The proof in Appendix B will show us that "opportunistic duplication scheme" will only improve the tradeoff with a $\log n$ factor.

The basic tradeoff of delay works similar to the former work [6], so we directly show upper bound of scheduling policy B under cluster dense regime for simplification.

Theorem 6.4: Under cluster dense regime and scheduling policy B, let $D_{B}^{d}$ denote the mean delay averaged over all bits and let $\lambda_{B}^{d}$ be the throughput of each source-destination pair. The following upper bound holds,

$$
\left(\lambda_{B}^{d}\right)^{3} \leq O\left(\frac{\overline{D_{B}^{d}}}{n} \log ^{3} n\right)
$$

Theorem 6.3 and 6.4 show us the tradeoff of scheduling policy A and scheduling policy B respectively. We assume $\bar{D}_{A}^{d}=\bar{D}_{B}^{d}=\bar{D}^{d}$. Then the overall upper bound can be derived easily.

Theorem 6.5: Under cluster dense regime, let $\overline{D^{d}}$ denote the mean delay averaged over all bits and let $\lambda_{-}^{d}$ be the throughput of each source-destination pair. Assume $\bar{D}_{A}^{d}=\bar{D}_{B}^{d}=\bar{D}^{d}$. The following upper bound holds,

$$
\lambda^{d}=\max \left\{\lambda_{A}^{d}, \lambda_{B}^{d}\right\}
$$

Note that we use a kind of double scheduling policy. If $\lambda_{A}^{d} \geq \lambda_{B}^{d}$, we use scheduling policy A, and if $\lambda_{A}^{d}<\lambda_{B}^{d}$, we use scheduling policy B (values of $\lambda_{A}^{d}$ and $\lambda_{B}^{d}$ are decided by the formulation in the upper bound).

\section{Lower Bound of the Cluster Dense Regime}

We will construct an achievable lower bound for cluster dense regime in this section. As we use a kind of double scheduling policy, we divide our lower bound into two parts. Under a certain network condition $\left(m=n^{v}, R=n^{\beta}\right.$, and $\bar{D}^{d}=n^{d}$ are known), we choose lower bound $\mathrm{A}$, if $\lambda_{A}^{d} \geq \lambda_{B}^{d}$ and we choose lower bound $\mathrm{B}$, if $\lambda_{A}^{d}<\lambda_{B}^{d}$ (values of $\lambda_{A}^{d}$ and $\lambda_{B}^{d}$ are decided by the formulation in the upper bound).

\section{A. Lower bound A}

In lower bound $\mathrm{A}$, we divide our normal time slot into four subslots. The operations of each slot are shown below:

1) The nodes (source node and relays) create inter-cluster duplications via $u$ times broadcast.

2) Inter-cluster duplication is capture by range $l_{1 b}^{d}$ and transmitted to a node in $C_{d}$ via $h_{1 b}{ }^{d}$-hop unicast with single-hop transmission range $r_{b}^{h}$.

3) $R_{d b}^{d}$ intra-cluster duplications are created via broadcast.

4) Intra-cluster duplication is captured by range $l_{2 b}^{d}$ and transmitted to the destination via $h_{2 b}^{d}$-hop unicast with single-hop transmission range $r_{b}^{h}$. 
The scheduling parameters in our scheme are selected from Table IV, V, VI, and VII with different delay-tolerant conditions and system parameters. TDMA is applied as well, so each cell can have $1 / c_{5}^{d}$ fraction of time to transmit. Then we will describe our tradeoff achieving scheme and the network tessellation.

1) In the 1st subslot, we divide our whole network area into $\mathbb{T}_{1}^{d}=\left\lfloor n /\left(A_{d} \log n\right)\right\rfloor$ equal-area cells, where $A_{d}=$ $\min \left\{m R^{2} / n, R_{c b}^{d}\right\}$ is the broadcast area. Then each node in the cell is scheduled be active for at least $\lambda^{d} /\left(c_{5}^{d} \log n\right) \leq$ $1 /\left(c_{5}^{d} A_{d} \log n\right)$ fraction of subslot to broadcast. If any cell contain more than $c_{5}^{d} \log n / \lambda^{d}$ nodes, we denote it as Error $_{I A}^{d}$.

2) In the 2nd subslot, we divide our whole network area into $\mathbb{T}_{2}^{d}=\left\lfloor n /\left(\left(l_{1 b}^{d}\right)^{2} \log n\right)\right\rfloor$ equal-area cells. If a certain message $b$ is not capture by the cell within $\Theta\left(D^{d}\right)$ slots, we denote it as Error ${ }_{I I A}^{d}$. Then we further divide each cell into $\mathbb{T}_{3}^{d}=\left(h_{1 b}^{d}\right)^{2}$ equal-area mini cells. The message captured by the cell is transmitted to a node in its $C_{d}$ through mini cells via multihop unicast during this subslot (first along Xaxis, then along Y-axis). If the network falls to transmit the captured message to a node in its $C_{d}$ within a certain subslot, we denote it as Error ${ }_{I I I A}^{d}$.

$3)$ In the 3rd subslots, we divide our whole network area into $\mathbb{T}_{4}^{d}=\left\lfloor n^{2} /\left(m R^{2} R_{d b}^{d} \log n\right)\right\rfloor$ equal-area cells. Then each node in the cell is scheduled be active for at least $\lambda^{d} /\left(c_{5}^{d} \log n\right) \leq n /\left(c_{5}^{d} m R^{2} R_{d b}^{d} \log n\right)$ fraction of subslot to broadcast. If any cell contain more than $c_{5}^{d} \log n / \lambda^{d}$ nodes, we denote it as Error $_{I V A}^{d}$.

4) In the 4th subslots, we divide our whole network area into $\mathbb{T}_{5}^{d}=\left\lfloor n /\left(\left(l_{2 b}^{d}\right)^{2} \log n\right)\right\rfloor$ equal-area cells. If a certain message $b$ is not capture by the cell within $\Theta\left(D^{d}\right)$ slots, we denote it as Error $_{V A}^{d}$. Then we further divide each cell into $\mathbb{T}_{6}^{d}=\left(h_{2 b}{ }^{d}\right)^{2}$ equal-area mini cells. The message captured by the cell is transmitted to its destination through mini cells via multihop unicast during this subslot (first along $\mathrm{X}$-axis, then along Y-axis). If the network falls to transmit the captured message to its destination within a certain subslot, we denote it as Error $_{V I A}^{d}$.

Theorem 7.1: Error $_{I A}^{d} \rightarrow 0$, Error $_{I I A}^{d} \rightarrow 0, \cdots$, Error $_{V I A}^{d}$ $\rightarrow 0$ as $n \rightarrow \infty$. So our lower bound under cluster dense regime can achieve the per-node throughput of $\Theta\left(\lambda^{d} / \log n\right)$ with $\Theta\left(D^{d} \log n\right)$ delay.

Proof: Error $_{I A}^{d}$, Error $_{I I A}^{d}$, Error $_{I I I A}^{d}$ are similar to Error $_{I V A}^{d}$, Error $V_{V A}^{d}$, Error ${ }_{V I A}^{d}$, so we only prove the Error $_{I V A}^{d} \rightarrow 0$, Error $_{V A}^{d} \rightarrow 0$, and Error $_{V I A}^{d} \rightarrow 0$. All the values of scheduling parameters used in the following proof are chosen from Table VI and VII.

1) Error $_{I V A}^{d}$ :

We can solve the problem by considering the equivalent experiment. We throw $n$ balls into $\mathbb{T}_{4}^{d}$ urns. We denote the number of ball in each urn as $X_{I V}^{d}$. If $X_{I V}^{d}>$ $c_{5}^{d} \log n / \lambda^{d}$, Error $_{I V A}^{d}$ happens.

$$
\mathbb{E}\left[X_{I V}^{d}\right]=\frac{n}{\frac{n^{2}}{m R^{2} R_{d b}^{d} \log n}}=\frac{\log n}{\lambda^{d}}
$$

Using multiplicative form of Chernoff bound,

$$
\mathbf{P}\left[X_{I V}^{s}>\frac{2 \log n}{\lambda^{d}}\right]<\left(\frac{e}{4}\right)^{\frac{\log n}{\lambda^{d}}}<O\left(\frac{1}{n}\right)
$$

With $n \rightarrow \infty, \mathbf{P}\left[X_{I V}^{d}>\frac{2 \log n}{\lambda^{d}}\right] \rightarrow 0$, which indicates that $\mathbf{P}\left[\right.$ Error $\left._{I V A}^{d}\right] \rightarrow 0$, as $n \rightarrow \infty$.

2) Error $_{V A}^{d}$ :

We can solve the problem by considering the equivalent experiment. We throw $n \bar{D}^{d} \log n$ balls into $\left(R^{2} \mathbb{T}_{4}^{d} / n\right)\left(R^{2} \mathbb{T}_{5}^{d} / n\right)$ urns. We denote the number of ball in each urn as $X_{V}^{d}$. If $X_{V}^{d}=0$, Error $_{V A}^{d}$ happens.

$$
\mathbb{E}\left[X_{V}^{d}\right]=\frac{n \overline{D^{d}} \log n}{\frac{R^{2} n}{m R^{2} R_{d b}^{d} \log n} \frac{R^{2}}{\left(l_{2}^{d}\right)^{2} \log n}}=\log n
$$

Using multiplicative form of Chernoff bound,

$$
\mathbf{P}\left[X_{V}^{d}=0\right]<\mathbf{P}\left[X_{V}^{d}<\frac{\log n}{2}\right]<\left(\sqrt{\frac{2}{e}}\right)^{\log n}=O\left(\frac{1}{n}\right)
$$

With $n \rightarrow \infty, \mathbf{P}\left[X_{V}^{d}=0\right] \rightarrow 0$, which indicates that $\mathbf{P}\left[\operatorname{Error}_{V A}^{d}\right] \rightarrow 0$, as $n \rightarrow \infty$.

3) Error $_{V I A}^{d}$ :

In order to prevent the Error $_{V I A}^{d}$, three detailed issue should be ensured. a) Each mini cell should contain at least one node. b) Each relay shouldn't serve more than a certain source-destination pairs. c) Row or column of each mini cell should go through no more than a certain relays (here we only consider row, because the calculation of column is same as the calculation of row). Combining the b) and c), we obtain that row or column of each mini cell should go through no more than $\Theta\left(\log n / \lambda^{d}\right)$ messages These three issues can be transform into four equivalent experiment.

a) Experiment $A$ : We throw $n$ balls into $\mathbb{T}_{5}^{d} \mathbb{T}_{6}^{d}$ urns. We denote the number of ball in each urn as $X_{V I_{a}}^{d}$. If $X_{V I_{a}}^{d}=0$, Error $_{V I_{a}}^{d}$ happens.

$$
\mathbb{E}\left[X_{V I_{a}}^{d}\right]=\frac{n \overline{D^{d}} \log n}{\frac{n}{\left(l_{2}^{d}\right)^{2} \log n}\left(h_{2 b}^{d}\right)^{2}}=\log n
$$

Using multiplicative form of Chernoff bound,

$$
\begin{aligned}
\mathbf{P}\left[X_{V I_{a}}^{d}=0\right] & <\mathbf{P}\left[X_{V I_{a}}^{d}<\frac{\log n}{2}\right] \\
& <\left(\sqrt{\frac{2}{e}}\right)^{\log n}=O\left(\frac{1}{n}\right)
\end{aligned}
$$

With $n \rightarrow \infty, \mathbf{P}\left[X_{V I_{a}}^{d}=0\right] \rightarrow 0$, which indicates that $\mathbf{P}\left[\right.$ Error $\left._{V I_{a}}^{d}\right] \rightarrow 0$, as $n \rightarrow \infty$.

b) Experiment $B$ : We throw $n$ balls into $\mathbb{T}_{4}^{d} \mathbb{T}_{5}^{d}$ urns.

c) Experiment C: We throw $\mathbb{T}_{4}^{d}$ balls into $\sqrt{\mathbb{T}_{6}^{d}}$ urns.

d) Experiment $\mathrm{D}$ : The experiment $\mathrm{B}$ and $\mathrm{C}$ are created to calculate the number of messages go throw the row of each mini cell, so obtain a new experiment by combining them. We throw $n$ balls into $\mathbb{T}_{5}^{d} \sqrt{\mathbb{T}_{6}^{d}}$. 
We denote the number of ball in each urn as $X_{V I_{d}}^{d}$. If $X_{V I_{d}}^{d}>c_{7}^{d} \log n / \lambda^{d}$, Error ${ }_{V I_{d}}^{d}$ happens.

$$
\mathbb{E}\left[X_{V I_{d}}^{d}\right]=\frac{n}{\frac{n}{\left(l_{2 b}^{d}\right)^{2} \log n} h_{2 b}^{d}}=\frac{\log n}{\lambda^{d}}
$$

Using multiplicative form of Chernoff bound,

$$
\mathbf{P}\left[X_{V I_{d}}^{d}>c_{7}^{d} \frac{\log n}{\lambda^{d}}\right]<\left(\frac{e}{4}\right)^{\log n}=O\left(\frac{1}{n}\right)
$$

With $n \rightarrow \infty, \mathbf{P}\left[X_{V I_{d}}^{d}>c_{7}^{d} \log n / \lambda^{d}\right] \rightarrow 0$, which indicates that $\mathbf{P}\left[\right.$ Error $\left._{V I_{d}}^{d}\right] \rightarrow 0$, as $n \rightarrow \infty$ and $\mathbf{P}\left[\right.$ Error $\left._{V I A}^{d}\right] \rightarrow 0$, as $n \rightarrow \infty$.

\section{B. Lower bound $B$}

We show the optimal values of scheduling policy B in Table VIII. Then we solve the suspending question: whether the relays will be larger than the cluster number $m$ when $l_{b}^{d}>\Theta(R)$ ?

If this situation happens, two inequalities are induced, $R_{b}^{d}>$ $m=n^{v}$ and $l_{b}^{d}>R=n^{\beta}$. They are equal to $(1-d) / 3>v$ and $2(1-d) / 3>2 \beta$. Then we come to $1-d>v+2 \beta \geq 1$, which mean that this situation can only happens when $d<0$. So our basic tradeoff of delay stands well.

In lower bound $\mathrm{B}$, we divide our normal time slot into two subslots. The operations of each are shown below:

1) The nodes (source node and relays) create $R_{b}^{d}$ relays via $u$ times broadcast.

2) Relay is captured by range $l_{b}^{d}$ and transmitted to the destination via $h_{b}^{d}$-hop unicast with single-hop transmission range $r_{b}^{h}$.

The scheduling parameters in our scheme are selected from Table VIII with different delay-tolerant conditions and system parameters. TDMA is applied as well, so each cell can have $1 / c_{5}^{d}$ fraction of time to transmit. Then we will describe our tradeoff achieving scheme and the network tessellation.

1) In the 1st subslot, we divide our whole network area into $\mathbb{T}_{1}^{d}=\left\lfloor n /\left(A_{d} \log n\right)\right\rfloor$ equal-area cells, where $A_{d}=$ $\min \left\{m R^{2} / n, R_{b}^{d}\right\}$ is the broadcast area. Then each node in the cell is scheduled be active for at least $\lambda^{d} /\left(c_{5}^{d} \log n\right) \leq$ $1 /\left(c_{5}^{d} A_{d} \log n\right)$ fraction of subslot to broadcast. If any cell contain more than $c_{5}^{d} \log n / \lambda^{d}$ nodes, we denote it as Error ${ }_{I B}^{d}$.

2) In the 2nd subslots, we divide our whole network area into $\mathbb{T}_{2}^{d}=\left\lfloor n /\left(\left(l_{b}^{d}\right)^{2} \log n\right)\right\rfloor$ equal-area cells. If a certain message $b$ is not capture by the cell within $\Theta\left(D^{d}\right)$ slots, we denote it as Error $_{I I B}^{d}$. Then we further divide each cell into $\mathbb{T}_{3}^{d}=\left(h_{b}^{d}\right)^{2}$ equal-area mini cells. The message captured by the cell is transmitted to its destination through mini cells via multihop unicast during this subslot (first along $\mathrm{X}$-axis, then along Y-axis). If the network falls to transmit the captured message to its destination within a certain subslot, we denote it as $\operatorname{Error}_{I I I B}^{d}$.

Theorem 7.2: Error $_{I B}^{d} \rightarrow 0$, Error $_{I I B}^{d} \rightarrow 0$, Error $_{I I I B}^{d} \rightarrow 0$ as $n \rightarrow \infty$. So our lower bound under cluster dense regime can achieve the per-node throughput of $\Theta\left(\lambda^{d} / \log n\right)$ with $\Theta\left(D^{d} \log n\right)$ delay.
TABLE VIII: The order of the optimal values of the scheduling parameters under cluster dense regime and scheduling Policy B.

\begin{tabular}{|l|l|}
\hline$R_{b}^{d}:$ \# of relays & $\Theta\left(n^{(1-d) / 3}\right)$ \\
\hline$l_{b}^{d}:$ Capture Range & $\Theta\left(n^{(1-d) / 3} / \log ^{1 / 2} n\right)$ \\
\hline$h_{b}^{d}:$ \# of Hops & $\Theta\left(n^{(1-d) / 3} / \log n\right)$ \\
\hline$r_{b}^{h}:$ Transmission range of Each Hop & $\Theta\left(\log ^{\frac{1}{2}} n\right)$ \\
\hline
\end{tabular}

Proof: The proof is similar to lower bound A under cluster dense regime, so we omit it for simplification.

\section{UpPer Bound of the Cluster CRitical Regime}

We have solved the problem of the cluster sparse regime $(v+2 \beta<1)$ and the cluster dense regime $(v+2 \beta>1)$. We can derive the upper bound of the cluster critical regime $(v+2 \beta=1)$ from either result, which comes to the same bound. In this section, we derive the upper bound from the cluster sparse regime. Scheduling policy and upper bound are displayed, but the proof is omitted for simplification.

In cluster critical regime, we use double scheduling policy as well. The scheduling policy A is similar to the cluster sparse regime (opportunistic broadcast scheme is applied):

1) When $s$ and relays meet a cluster $C_{k}\left(k=1, \ldots, R_{c}{ }^{c}\right.$, where $R_{c}{ }^{c}$ is the number of inter-cluster duplication) not containing a relay, a relay will be created in $C_{k}$ via one-hop unicast.

2) If a relay meets $C_{d}$, a relay will be created in $C_{d}$ via one-hop unicast. If not, we come back to step 1).

3) Relays in $C_{d}$ create intra-cluster duplications in $C_{d}$ via broadcast $\left(R_{d}^{c}\right.$ denotes the overall number of intracluster duplications).

4) If one intra-cluster duplication is captured by the destination with range $l^{c}$, the message will be transmitted to destination via $h^{c}$-hop unicast. If not, we come back to step 3)

Scheduling policy B is similar to the scheduling policy B under cluster dense regime (opportunistic broadcast scheme is applied):

1) Nodes containing a certain message create relays via $k$ th broadcast with area $A_{c}\left(k=1, \ldots, \Theta(u) ; R_{b}{ }^{c}\right.$ denotes the overall relays).

2) If one relay is captured by the destination with range $l_{b}^{c}$, message will be transmitted to the node via $h_{b}^{c}$-hop unicast. If not, we come back to step 1).

For the upper bound of delay-throughput tradeoff, we directly show the following theorem.

Theorem 8.1: Under cluster critical regime, $D_{A}^{c}$ and $D_{B}^{c}$ denote the packet delay of scheduling policy A and scheduling policy B respectively. $\lambda_{A}^{c}$ and $\lambda_{B}^{c}$ denote the throughput of each source-destination pair of scheduling policy $A$ and scheduling policy B respectively. The following upper bound 
holds,

$$
\begin{cases}\left(\lambda_{A}^{c}\right)^{3} \leq O\left(\overline{\bar{D}_{A}^{c}} \log ^{3} n\right) & d \geq \frac{3}{2}-4 \beta \\ \lambda_{A}^{c} \leq O\left(\frac{\overline{D_{A}^{c}}}{m} \log ^{3} n\right) & d<\frac{3}{2}-4 \beta \\ \left(\lambda_{B}^{c}\right)^{3} \leq O\left(\frac{\overline{D_{B}^{c}}}{n} \log ^{3} n\right) & 0 \leq d \leq 1\end{cases}
$$

Let $\overline{D^{c}}$ denote the mean delay averaged over all bits and let $\lambda^{c}$ be the throughput of each source-destination pair. Assume $\overline{D_{A}^{c}}=\overline{D_{B}^{c}}=\overline{D^{c}}$.

$$
\lambda^{c}=\max \left\{\lambda_{A}^{c}, \lambda_{B}^{c}\right\}
$$

\section{LOWER Bound of the Cluster CRitical Regime}

As we use a kind of double scheduling policy, we divide our lower bound into two part. Under a certain network condition ( $m=n^{v}, R=n^{\beta}$, and $\bar{D}^{c}=n^{d}$ are known), we choose lower bound $\mathrm{A}$, if $\lambda_{A}^{c} \geq \lambda_{B}^{c}$ and we choose lower bound $\mathrm{B}$, if $\lambda_{A}^{c}<\lambda_{B}^{c}$ (values of $\lambda_{A}^{c}$ and $\lambda_{B}^{c}$ are decided by the formulation in the upper bound).

\section{A. lower bound A}

In lower bound $\mathrm{A}$, we will divide our normal time slot into three subslots. The operation of each slot are shown below:

1) The nodes (source node and relays) create inter-cluster duplications and the destination cluster $C_{d}$ receive data from inter-cluster duplication via one hop unicast with transmission range $r_{b}^{h}$.

2) $R_{d b}^{c}$ intra-cluster duplications are created via broadcast.

3) Intra-cluster duplication is captured by range $l_{b}^{c}$ and transmitted to the destination via $h_{b}^{c}$-hop unicast with single-hop transmission range $r_{b}^{h}$.

The scheduling parameters in our scheme are selected from Table II and Table III $(v+2 \beta=1)$ with different delaytolerant conditions and system parameters. TDMA is applied as well, so each cell can have $1 / c_{1}^{c}$ fraction of time to transmit. Then we will describe our tradeoff achieving scheme and the network tessellation.

1) In the 1st subslot, we divide our whole network area into $\mathbb{T}_{1}^{c}=\lfloor n / \log n\rfloor$ cells. Then each node in the cell is scheduled to transmit at least $\left\lfloor 1 / \lambda^{c}\right\rfloor$ messages with $\lambda^{c} /\left(c_{1}^{c} \log n\right)$ length for a certain subslot, which is on the basis of that the network can sustain $\Theta(1)$ per-node throughput of inter-cluster communication. If network falls to sustain $\Theta(1)$ per-node throughput of inter-cluster communication, we denote it as Error $_{I A}^{c}$. And if a message cannot be sent to its $C_{d}$ within $\Theta\left(D^{c}\right)$ time slots, we denote it as Error ${ }_{I I A}^{c}$.

2) In the 2nd subslots, we divide our whole network area into $\mathbb{T}_{2}^{c}=\left\lfloor n /\left(R_{d b}^{c} \log n\right)\right\rfloor$ cells. Then each node in the cell is scheduled be active for at least $\lambda^{c} /\left(c_{1}^{c} \log n\right) \leq$ $1 /\left(c_{1}^{c} R_{d b}^{c} \log n\right)$ fraction of subslot to broadcast. If any cell contain more than $c_{1}^{c} \log n / \lambda^{c}$ nodes, we denote it as Error $_{I I I A}^{c}$.

3) In the 3rd subslots, we divide our whole network area into $\mathbb{T}_{3}^{d}=\left\lfloor n /\left(\left(l_{b}^{c}\right)^{2} \log n\right)\right\rfloor$ cells. If a certain message $b$ is not capture by the cell within $\Theta\left(D^{c}\right)$ slots, we denote it as Error ${ }_{I V A}^{c}$. Then we further divide each cell into $\mathbb{T}_{4}^{d}=\left(h_{b}^{c}\right)^{2}$ mini cells. The message captured by the cell is transmitted to its destination through mini cells via multihop unicast during this subslot (first along $\mathrm{X}$-axis, then along Y-axis). If the network falls to transmit the captured message to its destination within a certain subslot, we denote it as $\operatorname{Error}_{V A}^{c}$.

Theorem 9.1: Error $_{I A}^{c} \rightarrow$ 0,Error IIA $_{\text {IA }} \rightarrow 0, \cdots$, Error $_{V A}^{c}$ $\rightarrow 0$ as $n \rightarrow \infty$, so our lower bound can achieve the throughput of $\Theta\left(\lambda^{c} / \log ^{2} n\right)$ with $\Theta\left(D^{d} \log ^{2} n\right)$ delay.

Proof: The proof is similar to case under cluster sparse regime ans cluster dense regime, so we omit it for simplification.

\section{B. Lower bound B}

In lower bound $\mathrm{B}$, we divide our normal time slot into two subslots. The operation of each are shown below:

1) Nodes containing a certain message create relays via $k$ th broadcast with area $\Theta(\log n)\left(k=1, \ldots, \Theta(u) ; R_{b}^{c}\right.$ denotes the overall relays).

2) Relay is captured by range $l_{b}^{c}$ and transmitted to the destination via $h_{b}^{c}$-hop unicast with single-hop transmission range $r_{b}^{h}$.

The scheduling parameters in our scheme are selected from Table VIII $(v+2 \beta=1)$ with different delay-tolerant conditions and system parameters. TDMA is applied as well, so each cell can have $1 / c_{1}^{c}$ fraction of time to transmit. Then we will describe our tradeoff achieving scheme and the network tessellation.

1) In the 1st subslot, we divide our whole network area into $\mathbb{T}_{1}^{c}=\lfloor n / \log n\rfloor$ equal-area cells. Then each node in the cell is scheduled be active for at least $\lambda^{c} /\left(c_{1}^{c} \log n\right) \leq 1 /\left(c_{1}^{c} \log n\right)$ fraction of subslot to broadcast. If any cell contain more than $c_{1}^{c} \log n / \lambda^{c}$ nodes, we denote it as Error $_{I B}^{c}$.

2) In the 2nd subslots, we divide our whole network area into $\mathbb{T}_{2}^{c}=\left\lfloor n /\left(\left(l_{b}^{c}\right)^{2} \log n\right)\right\rfloor$ equal-area cells. If a certain message $b$ is not capture by the cell within $\Theta\left(D^{c}\right)$ slots, we denote it as Error $_{I I B}^{c}$. Then we further divide each cell into $\mathbb{T}_{3}^{c}=\left(h_{b}^{c}\right)^{2}$ equal-area mini cells. The message captured by the cell is transmitted to its destination through mini cells via multihop unicast during this subslot (first along $\mathrm{X}$-axis, then along Y-axis). If the network falls to transmit the captured message to its destination within a certain subslot, we denote it as $\operatorname{Error}_{I I I B}^{c}$.

Theorem 9.2: Error $_{I B}^{c} \rightarrow$ 0,Error IIB $^{c} \rightarrow 0$, Error $_{I I I B}^{c} \rightarrow 0$ as $n \rightarrow \infty$. So our lower bound under cluster dense regime can achieve the per-node throughput of $\Theta\left(\lambda^{c} / \log n\right)$ with $\Theta\left(D^{c} \log n\right)$ delay.

Proof: The proof is similar to lower bound B under cluster dense regime, so we omit it for simplification.

\section{DISCUSSION}

We have studied the delay-throughput tradeoff of correlated mobility for all sub-cases: the cluster sparse regime, the cluster dense regime, and the cluster critical regime. The impact of node correlation to the per-node throughput and packet delay will be discussed in this section. 


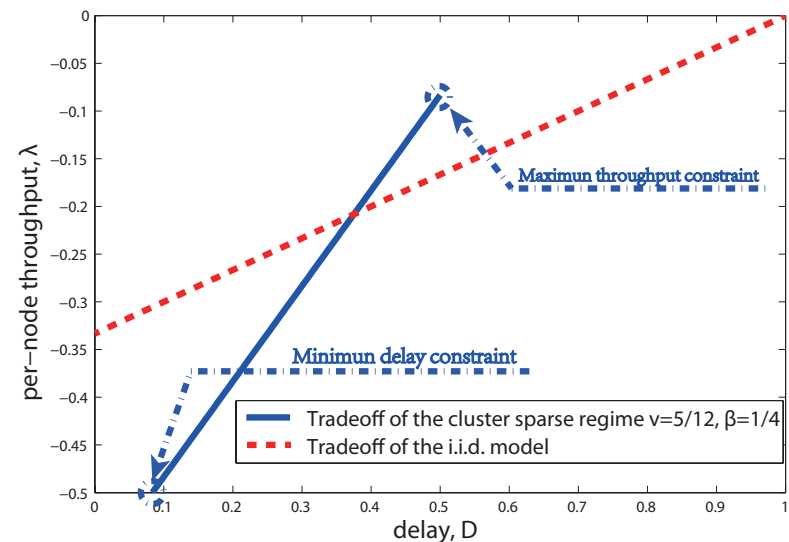

Fig. 6: Tradeoff of the cluster sparse regime when $v=5 / 12$, $\beta=1 / 4$; Tradeoff of the i.i.d. model (The marks on the axes represent the orders asymptotically in $\mathrm{n}$ ).

Under the cluster sparse regime, the nodes mobile with strong correlation (i.e., nodes form a few number of clusters or nodes in each cluster mobile within a small range). Then clusters in the network suffer a certain degree of disconnectivity, which restricts the maximum per-node throughput $\left(m R^{2} / n\right)$ and minimum packet delay $\left(n /\left(m R^{2}\right)\right)$. These two constrains greatly degrade the performance of the network; we can neither obtain a high per-node throughput nor a low packet delay, so the application will be limited.

Double scheduling policy is not applied here because of the strong node correlation and network disconnectivity. The scheduling policy $\mathrm{B}$ in double scheduling policy is a kind of direct relay-capture mechanism; we directly forward the message to the capture range of destination. Assume the number of relays in policy B is same as the number of intercluster duplications and intra-cluster duplications in policy A (the original policy applied by cluster sparse regime) respectively. Then the delay needed for policy B is larger than the delay needed for policy A when two policies can sustain the same asymptotic throughput, because the capture range of destination in policy B cannot be larger than $\Theta(R)$ or the capture range will exist disconnected area.

Fig. 6 is the delay-throughput tradeoff of the cluster sparse regime when $v=5 / 12, \beta=1 / 4$. We can see that there still exist a certain range for us to tradeoff. When we design the network to perform with high throughput, the tradeoff will be better than the optimal tradeoff of i.i.d. slow mobility model [6]. But our tradeoff become bad if the network is design to perform with low delay.

Under the cluster dense regime, the mobility of nodes show weak correlation and nodes within different clusters meet each other frequently. Obviously, network won't suffer the disconnectivity, so the constraints for throughput and delay doesn't exist any more. But extremely weak correlation may not improve the tradeoff performance. Too many clusters and too large area that each cluster covers lead the nodes within the

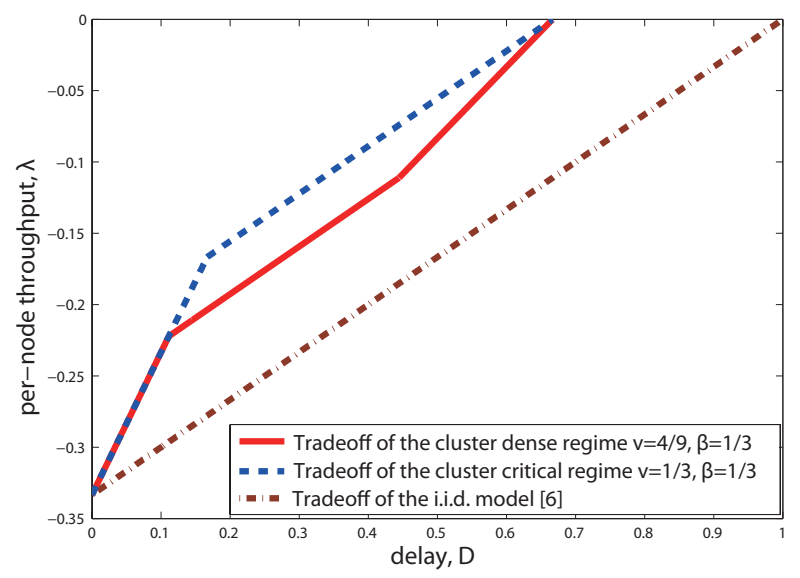

Fig. 7: Tradeoff of the cluster dense regime when $v=4 / 9$, $\beta=1 / 3$; Tradeoff of the cluster critical regime when $v=1 / 3$, $\beta=1 / 3$; Tradeoff of the i.i.d. model (The marks on the axes represent the orders asymptotically in $n$ ).

same cluster meet rarely, which drops the delay performance.

Fig. 7 contains the delay-throughput tradeoff of the cluster dense regime when $v=4 / 9, \beta=1 / 3$. It indicates that the tradeoff of correlated mobility model under cluster dense regime is much better than (at least equal to) the tradeoff of i.i.d. slow mobility model. We can design a network with specific tradeoff by controlling the system parameters $m=n^{v}$ and $R=n^{\beta}$.

Under the cluster critical regime, the mobility of nodes show medium correlation, which helps cluster critical regime being the best-performance regime among three sub-cases. From Fig. 7 , we can see that the delay-throughput tradeoff of the cluster critical regime perform better than the tradeoff of the cluster dense regime (broadcast under cluster critical regime uses less radio resource), although these two tradeoffs have the same end points. A network with specific tradeoff can be designed by controlling the system parameters $m=n^{v}$ and $R=n^{\beta}$ as well.

Then we focus on the end points, the maximum throughput point and the minimum delay point of the tradeoff under cluster critical regime When $v=1 / 4(\beta=3 / 8)$, the network can achieve the best per-node throughput $\Theta\left(n^{-1 / 4}\right)$ under the condition that packet delay should remain $\Theta(1)$. When $v=1 / 2(\beta=1 / 4)$, the network can achieve the best packet delay $\Theta(\sqrt{n})$ under the condition that per-node throughput should remain $\Theta(1)$.

From Fig. 8, we know that extremely large or small system parameters $v$ and $\beta$, which indicates the extremely strong or weak node correlation, will drop the system performance.

\section{CONCLusion}

The correlated mobility has a huge impact on the delaythroughput tradeoff of mobile ad hoc network. In this paper, we give an overall picture of this mobility model and exploit the node correlation to achieve the delay-throughput tradeoff. 

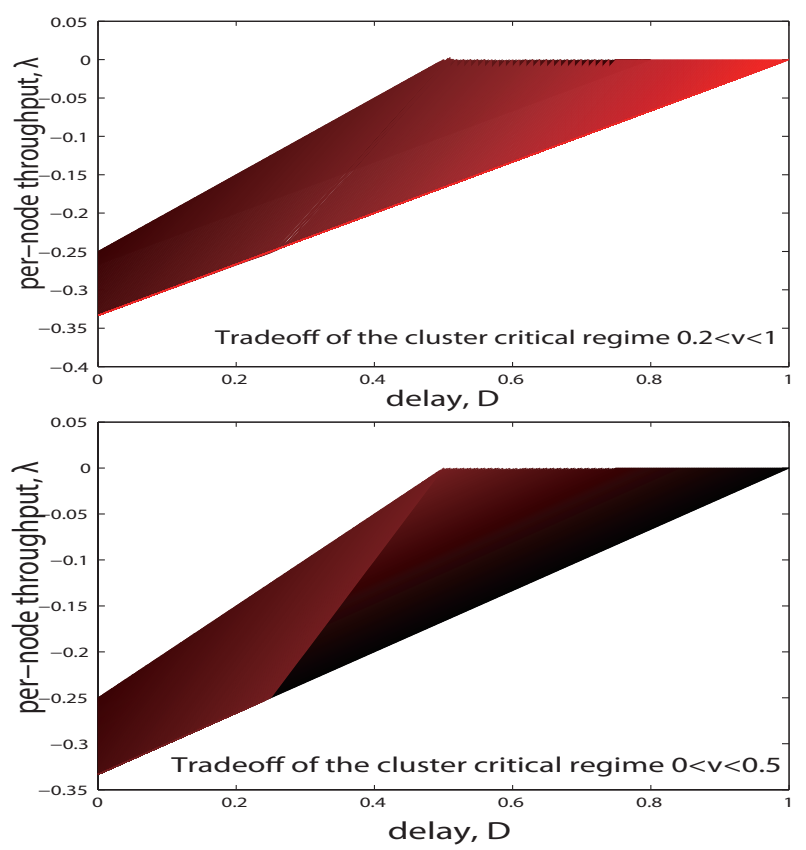

Fig. 8: Tradeoff of the cluster critical regime with various value of $m=n^{v}$ (The marks on the axes represent the orders asymptotically in $\mathrm{n}$ ). Brighter color means larger $m$ (upper fig.: $n^{0.2}<m<n$; lower fig.: $1<m<\sqrt{n}$ ).

We show that strong or extremely weak node correlation will not help improving or even drop the tradeoff performance. Our study reveal how the system parameters of node correlation improve the tradeoff performance and how to control the improvement through system and scheduling parameters. In the further study about the delay-throughput tradeoff of ad hoc mobile network, node correlation can be introduced to improve the network performance.

\section{REFERENCES}

[1] P. Gupta, P. R. Kumar, "The capacity of wireless networks," in IEEE Trans. on Info. Theory, Vol. 46, No. 2, pp. 388-404, March 2000.

[2] M. Franceschetti, O. Dousse, D.N.C. Tse and P.Thiran, "Closing the gap in the capacity of random wireless networks via percolation theory," in IEEE Trans. Inform. Theory, Vol. 53, No. 3, pp. 10091018, February 2007.

[3] M. Grossglauser and D. Tse, "Mobility Increase the Capacity of Ad Hoc Wireless Networks," in IEEE/ACM Transactions on Networking, vol.10, no. 4, August 2002.

[4] M. Neely and E. Modiano, "Capacity and delay tradeoffs for ad-hoc mobile networks," IEEE Trans. Inf. Theory, vol. 51, no. 6, pp. 1917-1937, June 2005.

[5] S. Toumpis and A. J. Goldsmith, "Large wireless networks under fading, mobility, and delay constraints," in Proc. IEEE Infocom, Hong Kong, China, 2004, vol. 1, pp. 619627.

[6] X. Lin, N. B. Shroff, "The Fundamental Capacity-Delay Tradeoff in Large Mobile Ad Hoc Networks," in Proc. MedHoc 2004.

[7] A. E. Gamal, J. Mammen, B. Prabhakar, and D. Shah, "Throughput-Delay Trade-off in Wireless Network," in Proc. IEEE Infocom, Hong Kong, China, March 2004.

[8] N. Bansal and Z. Liu, "Capacity, Delay and Mobility in Wireless Ad-Hoc Networks," in Proc.IEEE Infocom, San Francisco, CA, April 2003.

[9] E. Perevalov and R. Blum, "Even One-Dimensional Mobility Increases Ad Hoc Wireless Capacity," in ISIT 02, Lausanne, Switzerland, June 2002.
[10] M. Garetto, P. Giaccone, E. Leonardi, "Capacity Scaling in Delay Tolerant Networks with Heterogeneous Mobile Nodes," in Proc. ACM MobiHoc 2007.

[11] J. Mammen, D. Shah, "Throughput and Delay in Random Wireless Networks With Restricted Mobility," IEEE Trans. on Inf. Theory, 53(3), pp. 1108-1116, 2007.

[12] M. Garetto, P. Giaccone, E. Leonardi, "Capacity Scaling of Sparse Mobile Ad Hoc Networks," in Proc. Infocom, 2008.

[13] D. Ciullo, V. Martina, M. Garetto, E. Leonardi, "Impact of Correlated Mobility on Delay-Throughput Performance in Mobile Ad-Hoc Networks," in Proc. IEEE INFOCOM, March 2010.

[14] J. Yoon, B. D. Noble, M. Liu, M. Kim, "Building realistic mobility models from coarse-grained traces," in Proc. MobiSys, 2006.

[15] V.Naumov, R. Baumann, T.Gross, "An evaluation of inter-vehicle ad hoc networks based on realistic vehicular traces," in Proc. MobiSys, 2006.

[16] M. Musolesi, C. Mascolo, "Designing mobility models based on social network theory," Mob. Comput. Commun. Rev., 11(3), pp. 5970, 2007.

[17] P.Hui, J. Crowcroft, "Human mobility models and opportunistic communications system design," Phil. Trans. R. Soc. A, no. 366, pp. 2005-2016, 2008.

[18] X. Hong, M. Gerla, G. Pei and C. Chiang, "A group mobility model for ad hoc wireless networks," in Proc. ACM MSWiM 1999.

[19] M. Penrose, Random Geometric Graphs. Oxford University Press, 2003.

[20] R. Durrett, Theory and Examples, 2nd ed. Belmont, CA: Duxbury Press, 1996.

\section{APPENDIX A}

PROOF OF LEMMA 3.2

To simplify our proof process, some notation are needed. Under the cluster sparse regime, let $X_{i}^{s}(t)$ denote the position of node $i$ at time slot $t$. Let $b$ denote a bit message in our network. Let $t_{0 b}^{s}$ denote time when bit $b$ is generated. Let $l_{b}^{s \hat{(}}(t)$ denote the minimum distance form the edge of cluster containing duplication nodes (inter cluster duplication) to the edge of $C_{d}$ at time slot $t$, and $l_{b}^{s}(t)$ can be negative if inter cluster duplication and $C_{d}$ overlap. Let $\hat{L_{b}^{\hat{s}}(t)}=\max \left\{0, l_{b}^{s}(t)\right\}$. Let $R_{c b}^{s}(t)$ denote the number of inter cluster duplications at slot $t$. Let $t_{s b}^{s}$ denote the time when bit $b$ is captured by $C_{d}$.

We focus on the transmission of sending bit $b$ from source to its $C_{d}$ and $\mathbb{I}_{A}$ be the indicator function on set $\mathrm{A}$

$$
\begin{aligned}
\mathbb{E}\left[\frac{n}{\left(R+L_{b}^{\hat{s}(t))^{2}}\right]=}\right. & \mathbb{E}\left[\frac{n}{R^{2}} \mathbb{I}_{L_{b}^{s}(t) \leq 0}\right] \\
& +\mathbb{E}\left[\frac{n}{\left(R+l_{b}^{s(t))^{2}}\right.} \mathbb{I}_{L_{b}^{s}(t)>0}\right]
\end{aligned}
$$

Since the definition of expectation,

$$
\begin{aligned}
& \mathbb{E}\left[\frac{n}{\left(R+l_{b}^{s}(t)\right)^{2}} \mathbb{I}_{L_{b}^{s}(t)>0}\right] \\
= & \int_{0}^{\sqrt{n}} \frac{n}{(R+u)^{2}} d \mathbf{P}\left[l_{b}^{\hat{s}(t)) \leq u]}\right. \\
= & 1-\frac{n}{R^{2}} \mathbf{P}\left[l_{b}^{s(t)} \leq 0\right]+\int_{0}^{\sqrt{n}} \frac{2 n}{(R+u)^{3}} \mathbf{P}\left[l_{b}^{s}(t) \leq u\right] d u
\end{aligned}
$$


Therefore,

$$
\begin{aligned}
\mathbb{E}\left[\frac{n}{\left(R+L_{b}^{\hat{s}(t))^{2}}\right]}\right. & =1+\int_{R}^{\sqrt{n}} \frac{2 n}{u^{\prime 3}} \mathbf{P}\left[R+l_{b}^{s \hat{s}}(t) \leq u\right] d u^{\prime} \\
& =1+\int_{R}^{\sqrt{n}} 2 \pi R_{c b}^{s}(t) \frac{\left(R+u^{\prime}\right)^{2}}{u^{\prime 3}} d u^{\prime} \\
& \leq 1+6 \pi R_{c b}^{s}(t) \int_{R}^{\sqrt{n}} \frac{1}{u^{\prime}} d u^{\prime} \\
& =1+6 \pi R_{c b}^{s}(t) \log \frac{\sqrt{n}}{R} \\
& \leq 6 \pi R_{c b}^{s}(t) \log n
\end{aligned}
$$

We let

$W(t)=6 \pi \log n\left[t-t_{0 b}^{s}\right]-\sum_{t_{0}^{s}+1}^{t} \mathbb{E}\left[\frac{n}{\left(R+L_{b}^{\hat{s}}(t)\right)^{2} R_{c b}^{s}(t)} \mathbb{I}_{t=t_{s_{b}^{s}}^{s}}\right]$

Then

$$
\begin{aligned}
& \mathbb{E}[W(t)-W(t-1)] \\
= & 6 \pi \log n-\mathbb{E}\left[\frac{n}{\left(R+L_{b}^{\hat{s}}(t)\right)^{2} R_{c b}^{s}(t)} \mathbb{I}_{t=t_{s b}^{s}}\right] \\
\geq & 6 \pi \log n-\mathbb{E}\left[\frac{n}{\left(R+L_{b}^{\hat{s}(}(t)\right)^{2} R_{c b}^{s}(t)}\right] \\
\geq & 0
\end{aligned}
$$

which means that $W(t)$ is a sub-martingale. By the Optional Stopping Theorem [20]. We obtain

$$
6 \pi \log n \mathbb{E}\left[D_{I I_{b}^{s}}^{s}\right] \geq \mathbb{E}\left[\frac{n}{\left(R+\hat{L}_{b}^{\hat{s}}(t)\right)^{2} R_{c b}^{s}}\right]
$$

By Hölder's Inequality [20]

$$
\begin{aligned}
6 \pi \log n \mathbb{E}\left[D_{I I}^{s}\right] & \geq \frac{n}{\mathbb{E}^{2}\left[R+L_{b}^{\hat{s}}(t)\right] \mathbb{E}\left[R_{c b}^{s}\right]} \\
& \geq \frac{n}{\left(2 R+r_{b}^{s}\right)^{2} \mathbb{E}\left[R_{c b}^{s}\right]}
\end{aligned}
$$

Therefore,

$$
54 \pi \log n \mathbb{E}\left[D_{I I b}^{s}\right] \geq \frac{n}{R^{2} \mathbb{E}\left[R_{c b}^{s}\right]}
$$

The part for $D_{I I I b}^{s}$ is similar as Appendix B, so we directly give the result:

$$
8 \pi \log n \mathbb{E}\left[D_{I I I b}^{s}\right] \geq \frac{R^{2}}{\mathbb{E}\left[R_{d b}^{s}\right] \mathbb{E}\left[l_{b}^{s}+\frac{m R^{2}}{n^{2}}\right]^{2}}
$$

Inequality (34) and (35) lead to the Lemma 3.2 directly

\section{APPENDIX B}

Proof of Lemma 6.12

The main body of the proof is similar to Appendix A, so we will simplify our proof and pay attention on the different point. Let $b$ denote a bit message in our network. Let $t_{0}{ }^{d}$ denote time when bit $b$ is generated. Let $R_{b}^{d}(t)$ denote the relays at slot $t$. Let $l_{b}^{d \hat{(}(t)}$ denote the minimum distance among the relays to the destination at time slot $t$. Let $L_{b}^{\hat{d}}(t)=\max \left\{1 / n, l_{b}^{d \hat{(}}(t)\right\}$. Let $t_{s b}^{s}$ denote the time when bit $b$ is captured by the destination. $\mathbb{I}_{A}$ be the indicator function on set A.

$$
\begin{aligned}
\mathbb{E}\left[\frac{n}{\left(L_{b}^{\hat{d}(t))^{2}}\right]}\right. & =\mathbb{E}\left[n^{3} \mathbb{I}_{L_{b}^{d}(t) \leq \frac{1}{n}}\right]+\mathbb{E}\left[\frac{n}{\left(l_{b}^{d \hat{(}(t))^{2}}\right.} \mathbb{I}_{L_{b}^{d}(t)>\frac{1}{n}}\right] \\
& =1+\int_{\frac{1}{n}}^{\sqrt{n}} \frac{2 n}{u^{3}} \mathbf{P}\left[l_{b}^{s \hat{s}}(t) \leq u\right] d u \\
& \leq 1+\int_{\frac{1}{n}}^{\sqrt{n}} \frac{2 \pi n}{u^{3}} \frac{u^{2} R_{b}^{d}(t)}{n} d u \\
& \leq 8 \pi R_{b}^{d}(t) \log n
\end{aligned}
$$

We let

$W(t)=8 \pi \log n\left[t-t_{0 b}^{d}\right]-\sum_{t_{0} d+1}^{t} \mathbb{E}\left[\frac{n}{\left(L_{b}^{\hat{d}(}(t)\right)^{2} R_{b}^{d}(t)} \mathbb{I}_{t=t_{s_{b}^{d}}}\right]$

Then

$$
\begin{aligned}
& \mathbb{E}[W(t)-W(t-1)] \\
= & 8 \pi \log n-\mathbb{E}\left[\frac{n}{\left(L_{b}^{\hat{d}}(t)\right)^{2} R_{b}^{d}(t)} \mathbb{I}_{t=t_{s}^{d}}\right] \\
\geq & 8 \pi \log n-\mathbb{E}\left[\frac{n}{\left(L_{b}^{\hat{d}}(t)\right)^{2} R_{b}^{d}(t)}\right] \\
\geq & 0
\end{aligned}
$$

which means that $W(t)$ is a sub-martingale. By the Optional Stopping Theorem [20]. We obtain

$$
8 \pi \log n \mathbb{E}\left[D_{b}^{d}\right] \geq \mathbb{E}\left[\frac{n}{\left(L_{b}^{\hat{d}}(t)\right)^{2} R_{b}^{d}}\right]
$$

By Hölder's Inequality [20]

$$
8 \pi \log n \mathbb{E}\left[D_{b}^{d}\right] \geq \frac{n}{\mathbb{E}^{2}\left[l_{b}^{d}+\frac{1}{n}\right] \mathbb{E}\left[R_{b}^{d}\right]}
$$

